\title{
FRATTINI EXTENSIONS AND CLASS FIELD THEORY
}

\author{
TH. WEIGEL
}

\begin{abstract}
A. Brumer has shown that every profinite group of strict cohomological $p$-dimension 2 possesses a class field theory - the tautological class field theory. In particular, this result also applies to the universal $p$-Frattini extension $\tilde{G}_{p}$ of a finite group $G$. We use this fact in order to establish a class field theory for every $p$-Frattini extension $\pi: \tilde{G} \rightarrow G$ (Thm.A). The role of the class field module will be played by the $p$-Frattini module. The universal norms of this class field theory will carry important information about the $p$-Frattini extension $\pi: \tilde{G} \rightarrow G$. A detailled analysis will lead to a characterization of finite groups $G$ which have a $p$-Frattini extension $\pi: \tilde{G} \rightarrow G$ in which $\tilde{G}$ is a weakly-orientable $p$-Poincaré duality group of dimension 2 (Thm.B).

In section $\S 5$ we characterize the $p$-Frattini extensions $\pi_{A_{1}}: S l_{2}\left(\mathbb{Z}_{p}\right) \rightarrow$ $S l_{2}\left(\mathbb{F}_{p}\right), p \neq 2,3,5$, by some kind of localization technique. This answers a question posed by M.D.Fried and M.Jarden (Thm.C). It is quite likely that such an approach might also be successful for the characterization of the $p$ Frattini extensions $\pi_{D}: X_{D}\left(\mathbb{Z}_{p}\right) \rightarrow X\left(\mathbb{F}_{p}\right)$, where $X_{D}$ is the simple simplyconnected split $\mathbb{Z}$-Chevalley group scheme with Dynkin diagram $D$.
\end{abstract}

\section{INTRODUCTION}

Let $G$ be a finite group and let $p$ be a prime number. An extension of $G$ by a pro- $p$ group $A$

$$
1 \longrightarrow A \stackrel{\iota}{\longrightarrow} \tilde{G} \stackrel{\pi}{\longrightarrow} G \longrightarrow 1
$$

is called a $p$-Frattini extension, if $i m(\iota)$ is contained in the Frattini subgroup of $\tilde{G}$. The study of $p$-Frattini extensions of finite groups has a long history. W.Gaschütz (cf. [8]) showed that every finite group $G$ has a universal elementary $p$-abelian Frattini extension $\pi_{/ p}: \tilde{G}_{/ p} \rightarrow G$ which kernel is - considered as (left) $\mathbb{F}_{p}[G]$-module - isomorphic to $\Omega_{2}\left(G, \mathbb{F}_{p}\right)$, where $\Omega_{k}(G,-)=\Omega^{-k}(G,-)$ denotes the $k^{\text {th }}$-Heller translate in the category ${ }_{G} \bmod _{p}$ of finitely generated (left) $\mathbb{F}_{p}[G]$-modules. Based on this result J.Cossey, L.G.Kovács and O.H.Kegel [3] showed the existence of a universal $p$-Frattini cover $\pi_{p}: \tilde{G}_{p} \rightarrow G$. As the universal $p$-Frattini cover coincides with the minimal projective cover (cf. [6, Prop.20.33]), K.Gruenberg's theorem [7] implies that $\tilde{G}_{p}$ is of cohomological $p$-dimension less or equal to 1 , i.e, $c d_{p}\left(\tilde{G}_{p}\right) \leq 1$. In particular, $\operatorname{ker}\left(\pi_{p}\right)$ is a finitely generated free pro- $p$ group (cf. [12, $\S$ I.4.2, Cor.2]).

If $p$ divides the order of $G$, the profinite group $\tilde{G}_{p}$ is of strict cohomological $p$ dimension 2. For these groups A.Brumer [2] showed the existence of a tautological class field theory. The goal of this paper is to use this tautological class field theory for the group $\tilde{G}_{p}$ in order to obtain new result on $p$-Frattini extensions.

The most efficient way to establish a class field theory is to use the theory of cohomological Mackey-Functors. A. Dress introduced this notion in [4]. The

2000 Mathematics Subject Classification. Primary 20E18. 
exposition given by P.Webb in [15] will be particularly useful for our purpose, and therefore we will follow it closely as far as possible.

The following theorem can be seen as a "structure theorem for $p$-Frattini extensions", which combines W.Gaschütz theorem with the fact that the inflation mapping $H^{1}(\pi, S)$ is bijective for a $p$-Frattini extension $\pi$ as in (1.1) and an irreducible (left) $\mathbb{F}_{p}[G]$-module $S$ [16, Prop.3.1]. Its proof can be found in section 3.3 (cf. Thm.3.1, Cor.3.2).

Theorem A. Let $G$ be a finite group, let $p$ be a prime number and let $\pi: \tilde{G} \rightarrow G$ be a p-Frattini extension. Let $\mathcal{F}(\tilde{G})$ be the set of all open normal subgroups of $\tilde{G}$ being contained in $\operatorname{ker}(\pi)$. Then there exists a p-class field theory $(\mathbf{C}, \gamma)$ for $(\tilde{G}, \mathcal{F})$, i.e.,

(i) $\mathbf{C}$ is a cohomological $\mathcal{F}(\tilde{G})$-Mackey functor of type $H^{0}$ (this is a short form to say that it has Galois descent),

(ii) $\mathbf{C}_{U}=\Omega_{2}\left(\tilde{G} / U, \mathbb{Z}_{p}\right)$ for all $U \in \mathcal{F}(\tilde{G})$,

(iii) $\gamma: \mathbf{C} \rightarrow \mathbf{A} \mathbf{b}^{p}$ is a surjective morphism of cohomological $\mathcal{F}(\tilde{G})$-Mackey functors, where $\mathbf{A b}^{p}$ denotes the cohomological $\mathcal{F}(\tilde{G})$-Mackey functor of maximal p-abelian quotients (cf. §3.1),

(iv) for all $U, V \in \mathcal{F}(\tilde{G}), V \leq U, \gamma$ induces an isomorphism

$$
\mathbf{C}_{U} / i m\left(N_{V, U}^{\mathbf{C}}\right) \simeq(U / V)_{p}^{a b},
$$

(v) let $U, V, W \in \mathcal{F}(\tilde{G}), V, W \leq U$, such that $U / V$ and $U / W$ are abelian $p$ groups. Then $\operatorname{im}\left(N_{V, U}^{\mathrm{C}}\right)=i m\left(N_{W, U}^{\mathrm{C}}\right)$ implies $V=W$.

The class field theory $(\mathbf{C}, \gamma)$ has also two further properties one would usually require from a class field theory: (vi) There exists a canonical class $c \in$ nat $^{2}\left(\mathfrak{X}\left(\mathbb{Z}_{p}\right), \mathbf{C}\right)$, (vii) $H^{1}\left(\tilde{G} / V, \mathbf{C}_{V}\right)=H^{1}\left(U / V, \mathbf{C}_{V}\right)=0$ for all $U, V \in \mathcal{F}(\tilde{G})$, $V \leq U$ (cf. Rem.3.3). However, this will not be of importance for our purpose.

The kernel of $\gamma$ will be called the universal norms (of $\mathbf{C}$ ). Its analysis will finally enable us to characterize finite groups $G$ possessing a $p$-Frattini cover $\pi: \tilde{G} \rightarrow G$ in which $\tilde{G}$ is a weakly-orientable profinite $p$-Poincaré duality group of dimension 2 (cf. Cor.4.6). Here we call a profinite $p$-Poincaré duality group $\tilde{G}$ of dimension $d$ weakly-orientable, if $H^{d}\left(\tilde{G}, \mathbb{F}_{p} \llbracket \tilde{G} \rrbracket\right) \simeq \mathbb{F}_{p}$ is the trivial module.

Theorem B. Let $G$ be a finite group, and let $p$ be a prime number. Then the following are equivalent:

(i) There exist a p-Frattini extension $\pi: \tilde{G} \rightarrow G$, where $\tilde{G}$ is a profinite weaklyorientable p-Poincaré duality group of dimension 2.

(ii) There exists an injective map

$$
\alpha: \Omega^{1}\left(G, \mathbb{F}_{p}\right) \longrightarrow \Omega_{2}\left(G, \mathbb{F}_{p}\right)
$$

which is not an isomorphism.

Remark 1.1. Theorem B raises the following two questions: (1) For which finite groups $G$ and prime numbers $p$ does there exist an injective but not surjective map $\alpha: \Omega^{1}\left(G, \mathbb{F}_{p}\right) \longrightarrow \Omega_{2}\left(G, \mathbb{F}_{p}\right)$ ? (2) Provided such a mapping exists, how many isomorphism types of $p$-Frattini covers $\pi: \tilde{G} \rightarrow G$ exist, where $\tilde{G}$ is a weaklyorientable $p$-Poincaré duality group of dimension 2 ?

Unfortunately, we cannot say anything about the second question. Explicit computations using the work of K.Erdmann [5] show that for $q \equiv 3 \bmod 4$, such a mapping $\alpha$ exists for $G$ : $=P S l_{2}(q)$ and $p=2$ (cf. [16], [17]). However, it seems 
a very difficult problem to characterize or classify the tuples $(G, p)$ for which such a mapping exists.

Let $\mathfrak{S}_{p}(G)$ denote the set of isomorphism types of irreducible (left) $\mathbb{F}_{p}[G]$-modules, and let $\Delta \subseteq \mathfrak{S}_{p}(G)$ be a subset of $\mathfrak{S}_{p}(G)$. For short we call a $p$-Frattini extension $\pi: \tilde{G} \rightarrow G$ a $\Delta$-Frattini extension, if the isomorphism type of every $G$ composition factor of $\operatorname{ker}(\pi)$ is contained in $\Delta$. From the existence of the universal $p$-Frattini extension one deduces easily the existence of a universal $\Delta$-Frattini extension $\pi_{\Delta}: \tilde{G}_{\Delta} \rightarrow G$ (cf. $\S 5.2$ ). Obviously, $\tilde{G}_{\mathfrak{S}_{p}(G)}$ coincides with $\tilde{G}_{p}$, and $\tilde{G}_{\emptyset}$ coincides with $G$ itself. For our purpose it will be useful that the universal $\Delta$ Frattini extension can be charcterized by vanishing of second degree cohomology in a similar way as it is known for the universal $p$-Frattini extension (cf. Prop.5.1).

It is well-known that for $p \neq 3$, the extension

$$
\pi_{A_{1}}: S l_{2}\left(\mathbb{Z}_{p}\right) \longrightarrow S l_{2}\left(\mathbb{F}_{p}\right)
$$

is indeed a $p$-Frattini extension (cf. [18]). However, it remained an open problem to characterize the extension $\pi_{A_{1}}$ among all $p$-Frattini extension (cf. [6, Problem 20.40]).

For $p \neq 2,3$, M.Lazard's theorem implies that $S l_{2}\left(\mathbb{Z}_{p}\right)$ is an orientable $p$-Poincaré duality group of dimension 3 (cf. [13]). From this fact we will deduces the following characterization:

Theorem C. Let $p$ be a prime different from 2, 3 and 5 . Let $M_{k}, k=0, \ldots, p-1$, denote the simple $\mathbb{F}_{p}\left[S l_{2}\left(\mathbb{F}_{p}\right)\right]$-module of weight $k$ and $\mathbb{F}_{p}$-dimension $k+1$. Then for every subset $\Delta \subset \mathfrak{S}_{p}\left(\operatorname{Sl}_{2}\left(\mathbb{F}_{p}\right)\right)$ satisfying

(i) $\left[M_{2}\right] \in \Delta$,

(ii) $\left[M_{p-3}\right] \notin \Delta$,

the universal $\Delta$-Frattini extension $\pi_{\Delta}$ of $S l_{2}\left(\mathbb{F}_{p}\right)$ coincides with $\pi_{A_{1}}$, i.e., one has an isomorphism

$$
\phi: \widetilde{S} l_{2}\left(\mathbb{F}_{p}\right)_{\Delta} \longrightarrow S l_{2}\left(\mathbb{Z}_{p}\right)
$$

satisfying $\pi_{A_{1}} \circ \phi=\pi_{\Delta}$.

For a given Dynkin diagram $D$ let $X_{D}$ be the simple simply-connected $\mathbb{Z}$-Chevalley group scheme associated to $D$. It has been proved in [18] that apart from finitely many (more or less explicitly known) values of $(D, p)$,

$$
\pi_{D}: X_{D}\left(\mathbb{Z}_{p}\right) \longrightarrow X_{D}\left(\mathbb{F}_{p}\right)
$$

is a $p$-Frattini extension. Therefore, one wonders whether one can characterize $X_{D}\left(\mathbb{Z}_{p}\right)$ in a similar fashion as $S l_{2}\left(\mathbb{Z}_{p}\right)$ answering the problem raised in $[6$, Prob.20.40] in a wider context:

Question 1.2. Assume that $p$ is large with respect to the Coxeter number of $D$. Let $\mathfrak{L}_{D}\left(\mathbb{F}_{p}\right)$ denote the $\mathbb{F}_{p}$-Chevalley Lie algebra associated to $D$ considered as (left) $\mathbb{F}_{p}\left[X_{D}\left(\mathbb{F}_{p}\right)\right]$-module and put $\Delta_{D}:=\left\{\left[\mathfrak{L}_{D}\left(\mathbb{F}_{p}\right)\right]\right\}$. Are $\pi_{D}$ and $\pi_{\Delta_{D}}$ isomorphic pFrattini covers?

Remark 1.3. Proposition 5.1 shows that Question 1.2 is equivalent to the question whether

$$
H^{2}\left(X_{D}\left(\mathbb{Z}_{p}\right), \mathfrak{L}_{D}\left(\mathbb{F}_{p}\right)\right)=0
$$




\section{Cohomological Mackey functors}

2.1. Profinite modules of profinite groups. Let $p$ be a prime number, and let $\hat{G}$ be a profinite group. The completed $\mathbb{Z}_{p}$-group algebra of $\hat{G}$ is given by

$$
\mathbb{Z}_{p} \llbracket \hat{G} \rrbracket:=\lim _{\longleftarrow} \mathbb{Z}_{p}[\hat{G} / U],
$$

where the inverse system is running over all open normal subgroups of $\hat{G}$. By ${ }_{\hat{G}} \mathbf{p r f}{ }_{p}$ we denote the abelian category the objects of which are abelian pro- $p$ groups with continuous left $\hat{G}$-action. The morphisms from $M$ to $N, M, N \in o b\left({ }_{\hat{G}} \mathbf{p r f}{ }_{p}\right)$, are defined to be the continuous morphisms of profinite groups commuting with the action of $\hat{G}$. The abelian group of morphisms from $M$ to $N$ will be denoted by $\operatorname{Hom}_{\hat{G}}(M, N)$. This category can be identified with the full subcategory of the category of topological left $\mathbb{Z}_{p} \llbracket \hat{G} \rrbracket$-modules, the objects of which are also abelian pro- $p$ groups. It is well-known that ${ }_{G}$ prf ${ }_{p}$ has enough projectives, and in particular minimal projective covers. If $\hat{G}$ is the trivial group, then ${ }_{G} \mathbf{p r f}{ }_{p}$ coincides with the category of abelian pro- $p$ groups, which we will denote by $\operatorname{prf}_{p}$.

By ${ }_{G} \mathbf{p r f}{ }_{/ p}$ we denote the abelian category the objects of which are profinite $\mathbb{F}_{p}$-vector spaces with continuous left $\hat{G}$-action. It is a full subcategory of ${ }_{G} \mathbf{p r f} \mathbf{f}_{p}$, and objects can be considered as topological modules for the completed $\mathbb{F}_{p}$-group algebra

$$
\mathbb{F}_{p} \llbracket \hat{G} \rrbracket:=\varliminf_{U} \mathbb{F}_{p}[\hat{G} / U] .
$$

For further details the reader may wish to consult [2], [11] or [13].

2.2. Cohomological Mackey functors. There are several equivalent ways to define a cohomological Mackey functor. Here we will follow more or less the approach chosen by P.Webb (cf. $[15, \S 2])$.

Let $\hat{G}$ be a profinite group and let $\mathcal{N}$ be a set of open normal subgroups of $\hat{G}$. For short we call $\mathcal{N}$ a normal Mackey system, if $\mathcal{N}$ is closed with respect to products and intersections, and if $\bigcap_{U \in \mathcal{N}} U=1$.

Let $\mathcal{N}$ be a normal Mackey system of the profinite group $\hat{G}$. A cohomological $\mathcal{N}$ Mackey functor $\mathbf{X}$ with coefficients in $\mathbf{p r f}_{p}$ is a collection $\left(\mathbf{X}_{U}\right)_{U \in \mathcal{N}}$ of $\hat{G}$-modules $\mathbf{X}_{U} \in o b\left({ }_{\hat{G} / U} \mathbf{p r f}_{p}\right)$, together with two series of mappings $i_{U, V}^{\mathbf{X}}$ and $N_{V, U}^{\mathbf{X}}$ for $U, V \in$ $\mathcal{N}, V \leq U$, where

$$
\begin{array}{r}
i_{U, V}^{\mathbf{X}} \in \mathbf{H o m}_{\hat{G} / V}\left(\mathbf{X}_{U}, \mathbf{X}_{V}\right), \\
N_{V, U}^{\mathbf{X}} \in \mathbf{H o m}_{\hat{G} / V}\left(\mathbf{X}_{V}, \mathbf{X}_{U}\right),
\end{array}
$$

and which satisfy the following relations:

$$
\begin{aligned}
i_{U, U}^{\mathbf{X}} & =N_{U, U}^{\mathbf{X}}=i d_{\mathbf{X}_{U}} & & \text { for all } U \in \mathcal{N}, \\
i_{U, W}^{\mathbf{X}} & =i_{V, W}^{\mathbf{X}} \circ i_{U, V}^{\mathbf{X}} & & \text { for all } U, V, W \in \mathcal{N}, U \leq V \leq W, \\
N_{W, U}^{\mathbf{X}} & =N_{V, U}^{\mathbf{X}} \circ N_{W, V}^{\mathbf{X}} & & \text { for all } U, V, W \in \mathcal{N}, U \leq V \leq W, \\
i_{U V, V}^{\mathbf{X}} \circ N_{U, U V}^{\mathbf{X}} & =N_{U \cap V, V}^{\mathbf{X}} \circ i_{U, U \cap V}^{\mathbf{X}} & & \text { for all } U, V \in \mathcal{N}, \\
i_{U, V}^{\mathbf{X}} \circ N_{V, U}^{\mathbf{X}} & =\sum_{x \in U / V} x & & \text { for all } U, V \in \mathcal{N}, U \leq V, \\
N_{V, U}^{\mathbf{X}} \circ i_{U, V}^{\mathbf{X}} & =|U: V| . i d_{\mathbf{X}_{U}} & & \text { for all } U, V \in \mathcal{N}, U \leq V,
\end{aligned}
$$


The notation we have chosen is closer related to number theory than the one introduced in [15]. One can easily verify that the role of $I_{V}^{U}$ in [15] is played by $N_{V, U}^{\mathbf{X}}$, and $i_{U, V}^{\mathrm{X}}$ plays the role of $R_{V}^{U}$. Our axioms (2.3) and (2.4)-(2.6) are obviously equivalent to the axioms (0)-(5) in $[15, \S 2]$. The axioms $(2.7)$ and (2.8) are reformulating axiom (6) in [15], as we assumed that all open subgroups of $\hat{G}$ under consideration are normal in $\hat{G}$. Axiom (2.9) characterizes cohomological Mackey functors among all Mackey functors (cf. [15, $\S 7]$ ).

By $\mathfrak{C M}_{\mathcal{N}}\left(\hat{G}, \mathbf{p r f}_{p}\right)$ we denote the category of cohomological $\mathcal{N}$-Mackey functors of $\hat{G}$ with coefficients in $\operatorname{prf}_{p}$. A morphism between cohomological $\mathcal{N}$-Mackey functors $\eta: \mathbf{X} \rightarrow \mathbf{Y}$ is a sequence of mappings $\left(\eta_{U}\right)_{U \in \mathcal{N}}, \eta_{U} \in \mathbf{H o m}_{\hat{G} / U}\left(\mathbf{X}_{U}, \mathbf{Y}_{U}\right)$, for which the diagrams
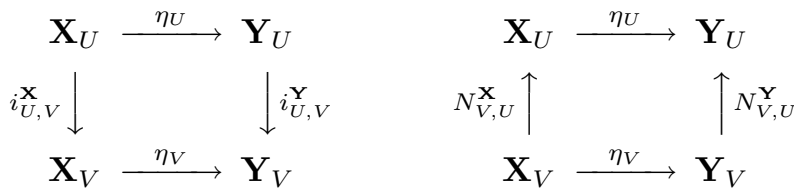

commute for all $U, V \in \mathcal{N}, V \leq U$. By $\operatorname{nat}(\mathbf{X}, \mathbf{Y})$ we denote the abelian group of morphisms of cohomological $\mathcal{N}$-Mackey functors from $\mathbf{X}$ to $\mathbf{Y}$.

Using the interpretation of $\mathfrak{C M}_{\mathcal{N}}\left(\hat{G}, \operatorname{prf}_{p}\right)$ as the category of additive $\mathbb{Z}_{p}$-linear functors from the category of $\hat{G}$-permutation modules of discrete $\hat{G}$-sets with isotropy group being contained in $\mathcal{N}$ to the category $\operatorname{prf}_{p}$ of abelian pro- $p$ groups (cf. [15, Prop.7.2]), one sees easily that $\mathfrak{C M}_{\mathcal{N}}\left(\hat{G}, \operatorname{prf}_{p}\right)$ is an abelian category. Kernels and cokernels are defined in the obvious way.

2.3. From cohomological Mackey functors to $\hat{G}$-modules and vice versa. Taking the inverse limit over the norm maps $N_{V, U}$ defines a covariant left exact functor

$$
\begin{gathered}
m: \mathfrak{C M}_{\mathcal{N}}\left(\hat{G}, \mathbf{p r f}_{p}\right) \longrightarrow{ }_{G} \mathbf{p r f}_{p}, \\
m(\mathbf{X}):=\varliminf_{U \in \mathcal{N}} \mathbf{X}_{U}, \quad \text { for } \mathbf{X} \in o b\left(\mathfrak{C M}_{\mathcal{N}}\left(\hat{G}, \operatorname{prf}_{p}\right)\right) .
\end{gathered}
$$

In case $\mathcal{N}$ contains a countable basis of neighbourhoods of $1 \in \hat{G}, \lim ^{1}$ vanishes, since all modules $\mathbf{X}_{U}$ are compact. Hence in this case $m$ is exact.

Let $M \in o b\left({ }_{G} \mathbf{p r f} \mathbf{f}_{p}\right)$ be an abelian pro- $p$ group with continuous left $\hat{G}$-action. For an open normal subgroup $U \in \mathcal{N}$ we denote by

$$
M_{U}:=\mathbb{Z}_{p}[\hat{G} / U] \hat{\otimes}_{\hat{G}} M=M / \operatorname{cl}(\langle(1-u) \cdot M \mid u \in U\rangle)
$$

the $U$-coinvariants of $M$. Here $\hat{\otimes}$ denotes the pro- $p$ tensor product as defined by A.Brumer (cf. $[2, \S 2]$ ), and $c l$ denotes the closure operation. The assignment $\mathfrak{X}(M)$ which assigns $U \in \mathcal{N}$ the $U$-coinvariants $\mathfrak{X}(M)_{U}:=M_{U}$ together with the natural map $N_{V, U}^{\mathfrak{X}(M)}: M_{V} \rightarrow M_{U}, V \leq U$, and the mapping $i_{U, V}^{\mathfrak{X}(M)}: M_{U} \rightarrow M_{V}, V \leq U$,

$$
i_{U, V}^{\mathfrak{X}(M)}\left(m+c l(\langle(1-u) \cdot M \mid u \in U\rangle):=\sum_{x \in V / U} x \cdot m+c l(\langle(1-v) \cdot M \mid v \in V\rangle),\right.
$$

defines a cohomological $\mathcal{N}$-Mackey functor $\mathfrak{X}(M) \in o b\left(\mathfrak{C M}_{\mathcal{N}}\left(\hat{G}, \operatorname{prf}_{p}\right)\right)$. It induces a covariant additive right exact functor

$$
\mathfrak{X}(-):{ }_{G} \operatorname{prf}_{p} \longrightarrow \mathfrak{C M}_{\mathcal{N}}\left(\hat{G}, \operatorname{prf}_{p}\right),
$$


which will be in general not exact. As we will see in the next subsection, the cohomological $\mathcal{N}$-Mackey functors obtained this way have a particular property which characterizes them.

2.4. Cohomology and homology of cohomological $\mathcal{N}$-Mackey functors. Let $\mathbf{X}$ be a cohomological $\mathcal{N}$-Mackey functor for $\hat{G}$ with coefficients in $\mathbf{p r f}_{p}$. For short we call $\mathbf{X} i$-injective, if all maps $i_{U, V}^{\mathbf{X}}, U, V \in \mathcal{N}, V \leq U$, are injective. Similarly, $\mathbf{X}$ is called $N$-surjective, if $N_{V, U}^{\mathbf{X}}$ is surjective for all $U, V \in \mathcal{N}, V \leq U$. if

Assume that $\mathbf{X} \in \operatorname{ob}\left(\mathfrak{C M}_{\mathcal{N}}\left(\hat{G}, \mathbf{p r f}_{p}\right)\right)$ is $i$-injective. Then we call $\mathbf{X}$ of type $H^{0}$,

$$
i m\left(i_{U, V}^{\mathbf{X}}\right)=\mathbf{X}_{V}^{U / V}
$$

for all $U, V \in \mathcal{N}, V \leq U$. Here $\mathbf{X}_{V}^{U / V}$ denotes the abelian group of $U / V$-fixed points on $\mathbf{X}_{V}$. Cohomological $\mathcal{N}$-Mackey functors of type $H^{0}$ are sometimes also called to have Galois descent. The $N$-surjective cohomological $\mathcal{N}$-Mackey functor is called of type $H_{0}$, if

$$
\operatorname{ker}\left(N_{V, U}^{\mathbf{X}}\right)=\sum_{x \in U / V}(x-1) \cdot \mathbf{X}_{V}
$$

for all $U, V \in \mathcal{N}, V \leq U$. From this definition it is straight forward, that a cohomological $\mathcal{N}$-Mackey functor is of type $H_{0}$, if and only if it is isomorphic to a functor $\mathfrak{X}(M)$ for some $M \in o b\left({ }_{G} \mathbf{p r f} f_{p}\right)$. The cohomological $\mathcal{N}$-Mackey functors being oy type $H_{0}$ are sometimes also called to have Galois codescent.

It is possible to interprete the definitions of being of type $H_{0}$ or of type $H^{0}$ in a more general homological context. For a cohomological $\mathcal{N}$-Mackey functor $\mathbf{X}$ we define for $U, V \in \mathcal{N}, V \leq U$,

$$
\begin{array}{ll}
\mathbf{k}^{0}(U / V, \mathbf{X}):=\operatorname{ker}\left(i_{U, V}^{\mathbf{X}}\right), & \mathbf{k}^{1}(U / V, \mathbf{X}):=\mathbf{X}_{V}^{U / V} / \operatorname{im}\left(i_{U, V}^{\mathbf{X}}\right), \\
2.18) & \\
\mathbf{c}_{0}(U / V, \mathbf{X}):=\operatorname{coker}\left(N_{V, U}^{\mathbf{X}}\right), & \mathbf{c}_{1}(U / V, \mathbf{X}):=\operatorname{ker}\left(N_{U, V}^{\mathbf{X}}\right) / \sum_{x \in U / V}(x-1) \mathbf{X}_{V} .
\end{array}
$$

Let $0 \rightarrow \mathbf{X} \rightarrow \mathbf{Y} \rightarrow \mathbf{Z} \rightarrow 0$ be a short exact sequence of cohomological $\mathcal{N}$-Mackey functors. Then the snake lemma implies that one has exact sequences

$$
\begin{gathered}
0 \rightarrow \mathbf{k}^{0}(U / V, \mathbf{X}) \rightarrow \mathbf{k}^{0}(U / V, \mathbf{Y}) \rightarrow \mathbf{k}^{0}(U / V, \mathbf{Z}) \ldots \\
\quad \rightarrow \mathbf{k}^{1}(U / V, \mathbf{X}) \rightarrow \mathbf{k}^{1}(U / V, \mathbf{Y}) \rightarrow \mathbf{k}^{1}(U / V, \mathbf{Z}), \\
\mathbf{c}_{1}(U / V, \mathbf{X}) \rightarrow \mathbf{c}_{1}(U / V, \mathbf{Y}) \rightarrow \mathbf{c}_{1}(U / V, \mathbf{Z}) \rightarrow \ldots \\
\mathbf{c}_{0}(U / V, \mathbf{X}) \rightarrow \mathbf{c}_{0}(U / V, \mathbf{Y}) \rightarrow \mathbf{c}_{0}(U / V, \mathbf{Z}) \rightarrow 0
\end{gathered}
$$

One can therefore think of $\mathbf{k}^{0 / 1}(U / V,-)$ as the 0 - and 1-dimensional section cohomology of cohomological $\mathcal{N}$-Mackey functors, and of $\mathbf{c}_{0 / 1}(U / V,-)$ as the 0 - and 1-dimensional section homology of cohomological $\mathcal{N}$-Mackey functors. It is possible to extend these functors to cohomological and homological functors, respectively. Since we will not make use of the higher derived functors we omit a detailed discussion here. However, we would like to remark, that these functors are not unrelated. 
Proposition 2.1. Let $\mathbf{X} \in \mathfrak{C M}_{\mathcal{N}}\left(\hat{G}, \operatorname{prf}_{p}\right)$ be a cohomological $\mathcal{N}$-Mackey functor and let $U, V \in \mathcal{N}, V \leq U$. Then one has an exact sequence of $\hat{G} / U$-modules

$$
\begin{aligned}
0 \longrightarrow & \mathbf{c}_{1}(U / V, \mathbf{X}) \stackrel{\alpha_{1}}{\longrightarrow} \hat{H}^{-1}\left(U / V, \mathbf{X}_{V}\right) \stackrel{\alpha_{2}}{\longrightarrow} \mathbf{k}^{0}(U / V, \mathbf{X}) \stackrel{\alpha_{3}}{\longrightarrow} \ldots \\
& \mathbf{c}_{0}(U / V, \mathbf{X}) \stackrel{\alpha_{4}}{\longrightarrow} \hat{H}^{0}\left(U / V, \mathbf{X}_{V}\right) \stackrel{\alpha_{5}}{\longrightarrow} \mathbf{k}^{1}(U / V, \mathbf{X}) \longrightarrow 0,
\end{aligned}
$$

where $\hat{H}^{\bullet}\left(U / V, \_\right)$denotes Tate cohomology.

Proof. The mapping $\alpha_{1}: \mathbf{c}_{1}(U / V, \mathbf{X}) \rightarrow \hat{H}^{-1}\left(U / V, \mathbf{X}_{V}\right)$ is clearly injective. Since $\alpha_{2}$ is induced by the norm map $N_{V, U}^{\mathbf{X}}$, one has

$$
k e r\left(\alpha_{2}\right)=\operatorname{ker}\left(N_{V, U}^{\mathbf{X}}\right) / \sum_{x \in U / V}(x-1) \mathbf{X}_{V}=i m\left(\alpha_{1}\right) .
$$

Furthermore, by axiom (2.9)

$$
\operatorname{ker}\left(\alpha_{3}\right)=\operatorname{ker}\left(i_{U, V}^{\mathbf{X}}\right) \cap i m\left(N_{V, U}\right)=N_{V, U}\left(\operatorname{ker}\left(\sum_{x \in U / V} x\right)\right)=i m\left(\alpha_{2}\right) .
$$

The mapping $\alpha_{4}$ is induced by $i_{U, V}^{\mathbf{X}}$. Hence

$$
k e r\left(\alpha_{4}\right)=\left(k e r\left(i_{U, V}^{\mathbf{X}}\right)+i m\left(N_{V, U}^{\mathbf{X}}\right)\right) / i m\left(N_{V, U}^{\mathbf{X}}\right)=i m\left(\alpha_{3}\right) .
$$

The mapping $\alpha_{5}$ is the canonical map and thus surjective. Furthermore,

$$
\operatorname{ker}\left(\alpha_{5}\right)=i m\left(i_{U, V}^{\mathbf{X}}\right) /\left(\sum_{x \in U / V} x\right) \cdot \mathbf{X}_{V}=i m\left(\alpha_{4}\right) .
$$

This yields the claim.

Remark 2.2. Let $\hat{G}$ be a finite cyclic group and let $\mathcal{N}:=\{1, \hat{G}\}$. Using an alternative approach for the definition of $\mathbf{c}_{\bullet}(\hat{G},-)$ and $\mathbf{k}^{\bullet}\left(\hat{G},,_{-}\right)$one sees that there exist connecting homomorphisms making the sequence

$$
\left(\mathbf{k}^{0}(\hat{G},-), \mathbf{k}^{1}(\hat{G},-), \mathbf{c}_{1}(\hat{G},-), \mathbf{c}_{0}(\hat{G},-)\right)
$$

a (co)homological functor. Let $M \in o b\left({ }_{\hat{G}} \mathbf{p r f}{ }_{p}\right)$ be a finitely generated $\mathbb{Z}_{p}[\hat{G}]$ module. Then (2.21) says that the Herbrand quotient (cf. [10, Kap.IV, §7])

$$
h(\hat{G}, M):=\frac{\left|\hat{H}^{0}(\hat{G}, M)\right|}{\left|\hat{H}^{-1}(\hat{G}, M)\right|}
$$

can be interpreted as a kind of multiplicative Euler characteristic, i.e., one has

$$
h(\hat{G}, M)=\frac{\left|\mathbf{c}_{0}(\hat{G}, \mathfrak{X}(M))\right| \cdot\left|\mathbf{k}^{1}(\hat{G}, \mathfrak{X}(M))\right|}{\left|\mathbf{c}_{1}(\hat{G}, \mathfrak{X}(M))\right| \cdot\left|\mathbf{k}^{0}(\hat{G}, \mathfrak{X}(M))\right|}=: \chi(\mathfrak{X}(M)) .
$$

For short we say that a cohomological $\mathcal{N}$-Mackey functor $\mathbf{X}$ is cohomologically trivial, if $\mathbf{X}$ is of type $H^{0}$ and $H_{0}$. From Proposition 2.1 follows that such a functor satisfies

$$
\hat{H}^{-1}\left(U / V, \mathbf{X}_{V}\right)=\hat{H}^{0}\left(U / V, \mathbf{X}_{V}\right)=0
$$

for all $U, V \in \mathcal{N}, V \leq U$.

Proposition 2.3. Let $P \in o b\left({ }_{\hat{G}} \mathbf{p r f} \mathbf{f}_{p}\right)$ be projective. Then for $V \in \mathcal{N}, \mathfrak{X}(P)_{V}$ (cf. 2.3) is a projective $\mathbb{Z}_{p}[\hat{G} / V]$-module. In particular, $\mathfrak{X}(P)$ is a cohomologically trivial cohomological $\mathcal{N}$-Mackey functor. 
Proof. The first statement follows from the fact that deflation from ${ }_{G} \mathbf{p r f}{ }_{p}$ to $\hat{G} / V \mathbf{p r f}_{p}$ is mapping projectives to projectives. Since restriction to closed subgroups is mapping projectives to projectives, it suffices to prove the second claim for $U=\hat{G}$. Since $\mathfrak{X}(P)$ is of type $H_{0}, \mathbf{c}_{0 / 1}(\hat{G} / V, \mathfrak{X}(P))=0$. As $P_{V} \in o b\left(\hat{G}_{\hat{G} / V} \mathbf{p r f}_{p}\right)$ is projective, $\hat{H}^{-1}\left(\hat{G} / V, P_{V}\right)=\hat{H}^{0}\left(\hat{G} / V, P_{V}\right)=0$. Hence Proposition 2.1 yields the claim.

\section{Class Field theories}

Throughout this section let $\hat{G}$ be a profinite group, and let $p$ be a prime number. We also assume that $\mathcal{N}$ is a normal Mackey system for $\hat{G}$.

For a finite group $G$ we denote by $\mathfrak{S}_{p}(G)$ the set of isomorphism types of irreducible (left) $\mathbb{F}_{p}[G]$-modules. For an irreducible $\mathbb{F}_{p}[G]$-module $S$ we use the symbol $[S] \in \mathfrak{S}_{p}(G)$ to denote its isomorphism type.

\subsection{The cohomological Mackey functors $\mathbf{A b}^{p}$ and $\mathbf{A} \mathbf{b}^{/ p}$. For $U \in \mathcal{N}$, let}

$$
\mathbf{A b}_{U}^{p}:=U_{p}^{a b}=U / \operatorname{cl}([U, U]) / O_{p^{\prime}}(U / c l([U, U])
$$

denote the largest continuous homomorphic image of $U$ which is an abelian pro- $p$ group. Here $[-,-]$ stands for the commutator subgroup, and $c l$ denotes the closure operation. Then for $U, V \in \mathcal{N}, V \leq U$, one has a canonical map $N_{V, U}^{\mathbf{A b}^{p}}: V_{p}^{a b} \rightarrow U_{p}^{a b}$. This map together with the transfer map (cf. [10, p.312])

$$
i_{U, V}^{\mathbf{A} \mathbf{b}^{p}}:=\operatorname{tr}_{V}^{U}: U_{p}^{a b} \rightarrow V_{p}^{a b}
$$

makes $\mathbf{A} \mathbf{b}^{p} \in \operatorname{ob}\left(\mathfrak{C} \mathfrak{M}_{\mathcal{N}}\left(\hat{G}, \operatorname{prf}_{p}\right)\right)$ a cohomological $\mathcal{N}$-Mackey functor. By $\mathbf{A} \mathbf{b}^{/ p}$ we denote its reduction modulo $p$, i.e., for $U \in \mathcal{N}$ one has

$$
\mathbf{A} \mathbf{b}_{U}^{/ p}:=U_{/ p}^{a b}=\mathbf{A} \mathbf{b}_{U}^{p} / p \cdot \mathbf{A} \mathbf{b}_{U}^{p}
$$

and the maps $i_{U, V}^{\mathbf{A b}^{\prime p}}$ and $N_{V, U}^{\mathbf{A b}^{\prime p}}, U, V \in \mathcal{N}, V \leq U$, are the maps induced from $i_{U, V}^{\mathbf{A} \mathbf{b}^{p}}$ and $N_{V, U}^{\mathbf{A} \mathbf{b}^{p}}$, respectively. It is obviously a cohomological $\mathcal{N}$-Mackey functor.

3.2. Weak $p$-class field theories. We define a weak p-class field theory $(\mathbf{X}, \eta)$ (for $(\hat{G}, \mathcal{N}))$ to be a cohomological $\mathcal{N}$-Mackey functor $\mathbf{X} \in o b\left(\mathfrak{C M}_{\mathcal{N}}\left(\hat{G}, \mathbf{p r f}_{p}\right)\right)$, together with a surjective morphism $\eta: \mathbf{X} \rightarrow \mathbf{A b}^{p}$ of cohomological $\mathcal{N}$-Mackey functors with the following properties:

(i) $\mathbf{X}$ is of type $H^{0}$,

(ii) $\mathbf{c}_{0}(U / V, \eta): \mathbf{c}_{0}(U / V, \mathbf{X}) \rightarrow(U / V)_{p}^{a b}$ is an isomorphism for all $U, V \in \mathcal{N}$, $V \leq U$.

The property (i) implies that $\mathbf{k}^{0 / 1}(U / V, \mathbf{X})=0$ for all $U, V \in \mathcal{N}, V \leq U$. In particular, one has an isomorphism $\mathbf{c}_{0}(U / V, \mathbf{X})=\hat{H}^{0}\left(U / V, \mathbf{X}_{V}\right)$. The property (ii) is one of the properties one would expect from a $p$-class field theory. However, in order to state the other property, one has also to require some structure on the normal Mackey system $\mathcal{N}$. 
3.3. $p$-Class field theories. For short we call a normal Mackey system $p$-closed, if it satisfies the following property: Assume that $W$ is an open normal subgroup of $\hat{G}$ which is contained in an open normal subgroup in $U \in \mathcal{N}$, such that $U / W$ is a finite abelian $p$-group. Then $W$ is also contained in $\mathcal{N}$.

Let $\mathcal{N}$ be a $p$-closed normal Mackey system of $\hat{G}$. Then we call the weak $p$-class field theory $(\mathbf{X}, \eta)$ a $p$-class field theory, if it satisfies additionally the following property:

(iii) Let $U \in \mathcal{N}$ and let $V, W \leq U$ be open and normal in $\hat{G}$, such that $U / V$ and $U / W$ are finite abelian $p$-groups. Assume that $i m\left(N_{V, U}^{\mathbf{X}}\right)=i m\left(N_{W, U}^{\mathbf{X}}\right)$. Then $V=W$.

In a similar fashion one defines a / $p$-class field theory: Let $\mathcal{N}$ be a $p$-closed normal Mackey system of $\hat{G}$. A cohomological $\mathcal{N}$-Mackey functor $\mathbf{X}$ together with a surjective morphism of $\mathcal{N}$-Mackey functors $\eta: \mathbf{X} \rightarrow \mathbf{A b}^{/ p}$ is called a $/ p$-class field theory, if the following properties hold:

(i) $\mathbf{X}$ is of type $H^{0}$,

(ii) $\mathbf{c}_{0}(U / V, \eta): \mathbf{c}_{0}(U / V, \mathbf{X}) \rightarrow(U / V)_{/ p}^{a b}$ is an isomorphism for all $U, V \in \mathcal{N}$, $V \leq U$

(iii) Let $U \in \mathcal{N}$ and let $V, W \leq U$ be open and normal in $\hat{G}$, such that $U / V$ and $U / W$ are finite elementary abelian $p$-groups. Assume that $i m\left(N_{V, U}^{\mathbf{X}}\right)=$ $i m\left(N_{W, U}^{\mathbf{X}}\right)$. Then $V=W$.

3.4. The $p$-Frattini class field theory and the $/ p$-Frattini class field theory. Let $G$ be a finite group, and let $\pi_{p}: \tilde{G}_{p} \rightarrow G$ denote its universal $p$-Frattini cover. We are considering the normal Mackey system

$$
\mathcal{F}:=\left\{U \leq \operatorname{ker}\left(\pi_{p}\right) \mid U \text { open and normal in } \tilde{G}_{p}\right\} .
$$

As $\operatorname{ker}\left(\pi_{p}\right)$ is a pro- $p$ group, it is obviously $p$-closed.

Let

$$
0 \longrightarrow P_{1} \stackrel{\delta}{\longrightarrow} P_{0} \stackrel{\varepsilon}{\longrightarrow} \mathbb{Z}_{p} \longrightarrow 0
$$

be a minimal projective resolution of the trivial $\mathbb{Z}_{p} \llbracket \tilde{G}_{p} \rrbracket$-module $\mathbb{Z}_{p}$ in $\tilde{G}_{p}$ prf $\mathbf{p}_{p}$. In particular, $\varepsilon: P_{0} \rightarrow \mathbb{Z}_{p}$ and $\delta^{\prime}: P_{1} \rightarrow \operatorname{ker}(\varepsilon)$ are minimal projective covers in $\tilde{G}_{p} \operatorname{prf}_{p}$.

Let $\mathfrak{S}_{p}(G)$ denote the set of isomorphism types of irreducible $\mathbb{F}_{p}[G]$-modules, and let $\tau_{S}: P_{S} \rightarrow S$ denote a minimal projective cover in ${\tilde{\sigma_{p}}}_{p} \mathbf{p r f}_{p},[S] \in \mathfrak{S}_{p}(G)$. As (3.5) is minimal, one has isomorphisms

$$
\operatorname{Hom}_{\tilde{G}_{p}}\left(P_{1}, S\right) \simeq H^{1}\left(\tilde{G}_{p}, S\right)
$$

for all $[S] \in \mathfrak{S}_{p}(G)$. In particular, $P_{1} \simeq \coprod_{[S] \in \mathfrak{S}_{p}(G)} P_{S}^{\mu_{S}}$, where

$$
\mu_{S}:=\frac{\operatorname{dim}_{\mathbb{F}_{p}}\left(H^{1}\left(\tilde{G}_{p}, S\right)\right)}{\operatorname{dim}_{\mathbb{F}_{p}}\left(\operatorname{End}_{G}(S)\right)} .
$$

Let $U \in \mathcal{F}$. As $-U$ is right exact, one has an exact sequence

$$
\left(P_{1}\right)_{U} \stackrel{\delta_{U}}{\longrightarrow}\left(P_{0}\right)_{U} \stackrel{\varepsilon_{U}}{\longrightarrow} \mathbb{Z}_{p} \longrightarrow 0
$$

As $\tilde{G}_{p} \rightarrow \tilde{G}_{p} / U$ is a $p$-Frattini extension, inflation induces isomorphisms

$$
H^{1}\left(\tilde{G}_{p}, S\right) \simeq H^{1}\left(\tilde{G}_{p} / U, S\right)
$$


for all $[S] \in \mathfrak{S}_{p}(G)$ (cf. [16, Prop.3.1]). This yields that

$$
H^{1}\left(\tilde{G}_{p} / U, S\right) \simeq \operatorname{Hom}_{\tilde{G}_{p} / U}\left(\left(P_{1}\right)_{U}, S\right)
$$

for all $[S] \in \mathfrak{S}_{p}(G)$, and from this one concludes easily that (3.8) is a partial minimal projective resolution. In particular, $\operatorname{ker}\left(\delta_{U}\right)=\Omega_{2}\left(\tilde{G}_{p} / U, \mathbb{Z}_{p}\right)$.

Let $\boldsymbol{\Omega}_{2}:=\operatorname{ker}(\mathfrak{X}(\delta))$. Then one has an exact sequence of cohomological $\mathcal{F}_{\text {- }}$ Mackey functors

$$
0 \longrightarrow \boldsymbol{\Omega}_{2} \longrightarrow \mathfrak{X}\left(P_{1}\right) \stackrel{\mathfrak{X}(\delta)}{\longrightarrow} \mathfrak{X}\left(P_{0}\right) \stackrel{\mathfrak{X}(\varepsilon)}{\longrightarrow} \mathfrak{X}\left(\mathbb{Z}_{p}\right) \longrightarrow 0,
$$

and $\Omega_{2, U}=\Omega_{2}\left(\tilde{G}_{p} / U, \mathbb{Z}_{p}\right)$.

From the Eckmann-Shapiro lemma for Tor. (cf. [13, Lemma 3.3.4]), and the canonical isomorphism $\mathbf{H}_{1}\left(U, \mathbb{Z}_{p}\right) \simeq U_{p}^{a b}=\mathbf{A b}_{U}^{p}$, where $\mathbf{H}_{\bullet}$ denotes homology as defined by A.Brumer (cf. $[2, \S 2]$ ), one obtains an isomorphism

$$
\eta: \boldsymbol{\Omega}_{2} \longrightarrow \mathbf{A b}^{p}
$$

of cohomological $\mathcal{F}$-Mackey functors.

By $\boldsymbol{\Omega}_{2}^{/ p}$ we denote the reduction $\bmod p$ of $\boldsymbol{\Omega}_{2}$, i.e., one has a short exact sequence in $\mathfrak{C M}_{\mathcal{F}}\left(\tilde{G}_{p}, \mathbf{p r f}_{p}\right)$

$$
0 \longrightarrow \boldsymbol{\Omega}_{2} \stackrel{p . i d}{\longrightarrow} \boldsymbol{\Omega}_{2} \longrightarrow \boldsymbol{\Omega}_{2}^{/ p} \longrightarrow 0 .
$$

By $\eta^{/ p}: \boldsymbol{\Omega}_{2}^{/ p} \rightarrow \mathbf{A b}^{/ p}$ we denote the induced isomorphism.

Theorem 3.1. Let $G$ be a finite group, $\pi_{p}: \tilde{G}_{p} \rightarrow G$ its universal $p$-Frattini cover, and let $\mathcal{F}$ be given as in (3.4).

(a) The tuple $\left(\boldsymbol{\Omega}_{2}, \eta\right)$ is a p-class field theory for $\left(\tilde{G}_{p}, \mathcal{F}\right)$.

(b) The tuple $\left(\boldsymbol{\Omega}_{2}^{/ p}, \eta^{/ p}\right)$ is a /p-class field theory for $\left(\tilde{G}_{p}, \mathcal{F}\right)$.

We call $\left(\boldsymbol{\Omega}_{2}, \eta\right)$ the $p$-Frattini class field theory for $\left(\tilde{G}_{p}, \mathcal{F}\right)$, and $\left(\boldsymbol{\Omega}_{2}^{/ p}, \eta^{/ p}\right)$ the $/ p$-Frattini class field theory for $\left(\tilde{G}_{p}, \mathcal{F}\right)$.

Proof. (a) One has to verify the axioms (i)-(iii). Axiom (ii) is obviously satisfied. Consider the short exact sequence

$$
0 \longrightarrow \boldsymbol{\Omega}_{2} \stackrel{\iota}{\longrightarrow} \mathfrak{X}\left(P_{1}\right) \longrightarrow \operatorname{coker}(\iota) \longrightarrow 0 .
$$

Since coker $(\iota)$ is a cohomological $\mathcal{F}$-subMackey functor of $\mathfrak{X}\left(P_{0}\right), \mathbf{k}^{0}(\operatorname{coker}(\iota))=0$ (cf. (2.19), Prop.2.3). The long exact sequence (2.19) applied to (3.14) and the cohomological triviality of $\mathfrak{X}\left(P_{0}\right)$ and $\mathfrak{X}\left(P_{1}\right)$ yields that $\boldsymbol{\Omega}_{2}$ is of type $H^{0}$. Hence axiom (i) is satisfied. It remains to verify (iii). We may assume that $p$ divides the order of the finite group $G$, since otherwise $\boldsymbol{\Omega}_{2}=0$, and there is nothing to prove. In this case $\tilde{G}_{p}$ is of cohomological $p$-dimension 1 , and thus of strict cohomological $p$-dimension 2 (cf. [12, §I.3.2]). In particular, by Brumer's theorem (cf. [2], [10, Kap.IV, §6, Aufg.6]) $\hat{G}$ possesses a tautological class field theory. Let $(\mathfrak{H}, \rho)$ denote its restriction to the Mackey system $\mathcal{F}$, i.e., $\mathfrak{H}_{U}=\mathbf{A b}_{U}^{p}$ and $\rho_{U}$ is the identity on $\mathbf{A} \mathbf{b}_{U}^{p}$. In particular $(\mathfrak{H}, \rho)$ and $\left(\boldsymbol{\Omega}_{2}, \eta\right)$ essentially coincide, i.e., one has a commutative diagram in $\mathfrak{C M}_{\mathcal{F}}\left(\tilde{G}_{p}, \operatorname{prf}_{p}\right)$

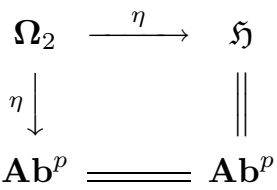


The property (iii) is well-known for $(\mathfrak{H}, \rho)$ (cf. [10, Kap.IV, Thm.6.7]). Thus it also holds for $\left(\boldsymbol{\Omega}_{2}, \eta\right)$.

(b) It suffices to prove that $\boldsymbol{\Omega}_{2}^{/ p}$ is of type $H^{0}$. The axiom (ii) is obvious, and axiom

(iii) follows from axiom (iii) for $\left(\boldsymbol{\Omega}_{2}, \eta\right)$.

Let $\mathfrak{X}\left(P_{0 / 1}\right)^{/ p}$ denote the reduction mod $p$ of $\mathfrak{X}\left(P_{0}\right)$ and $\mathfrak{X}\left(P_{1}\right)$, respectively. Then one has a short exact sequence

$$
0 \longrightarrow \boldsymbol{\Omega}_{2}^{/ p} \stackrel{\iota^{\prime p}}{\longrightarrow} \mathfrak{X}\left(P_{1}\right)^{/ p} \longrightarrow \operatorname{coker}\left(\iota^{/ p}\right) \longrightarrow 0,
$$

and $\operatorname{coker}\left(\iota^{/ p}\right)$ is a cohomological $\mathcal{F}$-sub Mackey functor of $\mathfrak{X}\left(P_{0}\right)^{/ p}$. From Proposition 2.1 one concludes that $\mathfrak{X}\left(P_{0}\right)^{/ p}$ and $\mathfrak{X}\left(P_{1}\right)^{/ p}$ are cohomologically trivial. Hence the long exact sequence (2.19) yields the claim.

Let $\pi: \tilde{G} \rightarrow G$ be any $p$-Frattini extension, finite or infinite. By universality, there exists a mapping $\tau: \tilde{G}_{p} \rightarrow \tilde{G}$, such that $\pi_{p}=\pi \circ \tau$. Since $\pi$ is a $p$-Frattini extension, $\tau$ is surjective. For short we put $N$ : $=\operatorname{ker}(\tau)$.

The morphism $\tau$ induces a canonical bijection of sets $\tau_{*}: \mathcal{F}_{N} \rightarrow \mathcal{F}(\tilde{G})$, where $\mathcal{F}$ is given as in (3.4) and

$$
\begin{aligned}
\mathcal{F}_{N}: & =\{U \in \mathcal{F} \mid N \leq U\}, \\
\mathcal{F}(\tilde{G}): & =\left\{U^{\prime} \leq \operatorname{ker}(\pi) \mid U^{\prime} \text { open and normal in } \tilde{G}\right\} .
\end{aligned}
$$

Let $\mathbf{C} \in o b\left(\mathfrak{C M} \mathfrak{F}_{\mathcal{F}(\tilde{G})}\left(\tilde{G}, \operatorname{prf}_{p}\right)\right)$ denote the cohomological $\mathcal{F}(\tilde{G})$-Mackey functor given by

$$
\mathbf{C}_{U}:=\boldsymbol{\Omega}_{2, \tau_{*}^{-1}(U)}, \quad U \in \mathcal{F}(\tilde{G})
$$

equipped with the obvious maps $i_{U, V}^{\mathrm{C}}, N_{V, U}^{\mathrm{C}}, U, V \in \mathcal{F}(\tilde{G}), V \leq U$. Let $\gamma: \mathbf{C} \rightarrow$ $\mathbf{A b}^{p}$ denote the morphism of $\mathcal{F}(\tilde{G})$-Mackey functors induced by $\eta$. In particular, $\gamma$ is surjective, but if $\tilde{G}$ does not coincide with the universal $p$-Frattini cover, $\gamma$ will not be an isomorphism.

Similarly, we define the reduction $\bmod p \mathbf{C}^{/ p}$ of $\mathbf{C}$, i.e., one has

$$
\mathbf{C}_{U}^{/ p}:=\Omega_{2, \tau_{*}^{-1}(U)}^{/ p}, \quad U \in \mathcal{F}(\tilde{G}),
$$

and by $\gamma^{/ p}: \mathbf{C}^{/ p} \rightarrow \mathbf{A b}^{/ p}$ we denote the surjective morphism induced by $\eta^{/ p}$. Again, apart from the case $\tilde{G} \simeq \tilde{G}_{p}, \gamma^{/ p}$ will not be surjective. From Theorem 3.1 one concludes:

Corollary 3.2. Let $G$ be a finite group, and let $\pi: \tilde{G} \rightarrow G$ be any p-Frattini extension. Then

(a) The tuple $(\mathbf{C}, \gamma)$ is a p-class field theory for $(\tilde{G}, \mathcal{F}(\tilde{G}))$.

(b) The tuple $\left(\mathbf{C}^{/ p}, \gamma^{/ p}\right)$ is a /p-class field theory for $(\tilde{G}, \mathcal{F}(\tilde{G}))$.

Remark 3.3. The definition of a $p$ or a $/ p$-class field theory we have given here is very much adapted to our main purpose, which is to prove Theorem B. Nevertheless, $\left(\boldsymbol{\Omega}_{2}, \eta\right)$ satisfies all class field theory axioms, which are usually required in number theory, i.e., using Tate cohomology one sees easily that for all $U, V \in \mathcal{F}, V \leq U$,

$$
H^{1}\left(U / V, \boldsymbol{\Omega}_{2, V}\right)=H^{1}\left(\tilde{G}_{p} / V, \boldsymbol{\Omega}_{2, V}\right)=0 .
$$

Moreover, (3.11) defines a canonical class $c \in \operatorname{nat}^{2}\left(\mathfrak{X}\left(\mathbb{Z}_{p}\right), \boldsymbol{\Omega}_{2}\right)$, where nat ${ }^{\bullet}(-,-)$ denote the derived functors of nat $(-,-)$ (cf. [9, Chap.XII]). This also applies to the $p$-class field theory $(\mathbf{C}, \gamma)$ defined for any $p$-Frattini cover $\pi: \tilde{G} \rightarrow G$. However, 
TH. WEIGEL

as the reader might verify by himself, (3.20) does not hold for the $/ p$-class field theories $\left(\boldsymbol{\Omega}_{2}^{/ p}, \eta^{/ p}\right)$ or $\left(\mathbf{C}^{/ p}, \gamma^{/ p}\right)$. Nevertheless, as we will see in the next section, these are the class field theories which are easiest to deal with.

\section{4. p-Poincaré DUALity GRoups of Dimension 2 AS p-Frattini EXTENSIONS}

Throughout this section we assume that $G$ is a finite group, and that $\pi: \tilde{G} \rightarrow G$ is a $p$-Frattini extension. By

$$
\begin{gathered}
P_{1} \stackrel{\delta}{\longrightarrow} P_{0} \stackrel{\varepsilon}{\longrightarrow} \mathbb{Z}_{p}, \\
Q_{1} \stackrel{\delta^{\prime p}}{\longrightarrow} P_{0} \stackrel{\varepsilon^{/ p}}{\longrightarrow} \mathbb{F}_{p}
\end{gathered}
$$

we denote partial minimal projective resolutions in $\tilde{G}_{\tilde{G}} \mathbf{p r f}_{p}$ and $\tilde{G}_{\tilde{G}} \mathbf{p r f}{ }_{/ p}$, respectively.

4.1. Universal norms. Let $\pi: \tilde{G} \rightarrow G$ be a $p$-Frattini extension, and let $(\mathbf{C}, \gamma)$ denote its $p$-Frattini class field theory. We call the cohomological $\mathcal{F}(\tilde{G})$-Mackey functor $\mathfrak{N}:=\operatorname{ker}(\gamma)$ the universal norms of $(\mathbf{C}, \gamma)$. Similarly, $\mathfrak{N}^{/ p}:=\operatorname{ker}\left(\gamma^{/ p}\right)$ will be called the universal norms of $\left(\mathbf{C}^{/ p}, \gamma^{/ p}\right)$. One has:

Proposition 4.1. Let $\pi: \tilde{G} \rightarrow G$ be a p-Frattini extension. Then:

(a) $\mathfrak{N}$ is $N$-surjective. Let $P_{1} \stackrel{\delta}{\longrightarrow} P_{0} \longrightarrow \mathbb{Z}_{p}$ be a partial minimal projective resolution of $\mathbb{Z}_{p}$ in ${ }_{\tilde{G}} \mathbf{p r f}_{p}$. Then $\operatorname{ker}(\delta) \simeq m(\mathfrak{N})$.

(b) $\mathfrak{N}^{\prime p}$ is $N$-surjective. Let $Q_{1} \stackrel{\delta}{\longrightarrow} Q_{0} \longrightarrow \mathbb{F}_{p}$ be a partial minimal projective resolution of $\mathbb{F}_{p}$ in $\tilde{G}_{\mathbf{G}} \mathbf{p r f}_{/ p}$. Then $\operatorname{ker}(\delta) \simeq m\left(\mathfrak{N}^{/ p}\right)$.

Proof. (a) For simplicity let us assume that $\iota: \mathfrak{N} \rightarrow \mathbf{C}$ is given by inclusion. Let $\left\{U_{k}\right\}_{k \in \mathbb{N}} \subseteq \mathcal{F}(\tilde{G})$ be a linearly ordered basis of neighbourhoods of $1 \in \tilde{G}$. We have to show that for $x \in \bigcap_{m \geq n} i m\left(N_{U_{m}, U_{n}}^{\mathrm{C}}\right)$, there exists a sequence $\left(y_{k}\right)_{k \in \mathbb{N}_{0}}$, $y_{k} \in \mathbf{C}_{U_{n+k}}$, such that $y_{0}=x$ and $y_{k}=N_{U_{n+k+1}, U_{n+k}}\left(y_{k+1}\right)$.

Let $Z:=\prod_{k \in \mathbb{N}_{0}} \mathbf{C}_{U_{n+k}}$. Then $Z$ is compact by Tychonoff's theorem. Let

$$
Z_{x, r}:=\left\{\left(z_{k}\right)_{k \in \mathbb{N}_{0}} \in Z \mid z_{0}=x, N_{U_{k+1}, U_{k}}\left(z_{k+1}\right)=z_{k} \text { for all } k \leq r .\right\} .
$$

Then $Z_{x, r+1} \subseteq Z_{x, r}$ and all sets $Z_{x, r}$ are closed. By definition, any finite intersection of sets $Z_{x, r}$ is non-empty. Hence $Z_{x, \infty}:=\bigcap_{r \in \mathbb{N}} Z_{x, r}$ is non-empty. Any element $\left(y_{k}\right)_{k \in \mathbb{N}_{0}} \in Z_{x, \infty}$ will have the desired property.

By construction, $\operatorname{ker}(\mathfrak{X}(\delta))=\mathbf{C}$. Moreover, one has a short exact sequence of $\mathcal{F}(\tilde{G})$-Mackey functors $0 \rightarrow \mathfrak{N} \rightarrow \mathbf{C} \rightarrow \mathbf{A} \mathbf{b}^{p} \rightarrow 0$. Obviously, $m\left(\mathbf{A} \mathbf{b}^{p}\right)=0$. Thus the claim follows from the exactness of $m$. The assertion (b) follows by a similar argument.

4.2. Weakly oriented $p$-Poincaré duality groups. Let $\hat{G}$ be a profinite group of cohomological $p$-dimension $d, d \in \mathbb{N}$. Then $\hat{G}$ is called a $p$-Poincaré duality group of dimension $d$, if

(i) for every finite discrete left $\hat{G}$-module of $p$-power order $X$ and for all $k \in \mathbb{N}_{0}$ one has

$$
\left|H^{k}(\hat{G}, X)\right|<\infty,
$$

(ii) the $p$-dualizing module $\mathbb{I}_{\hat{G}, p}$ of $\hat{G}$ is isomorphic to $\mathbb{Q}_{p} / \mathbb{Z}_{p}$ as abelian group, 
(iii) for every finite discrete left $\hat{G}$-module of $p$-power order $X$, cup-product induces a non-degenerate pairing

$$
H^{k}\left(\hat{G}, X^{\prime}\right) \times H^{d-k}(\hat{G}, X) \stackrel{H^{d}\left(e v_{X}\right) \circ(. \cup .)}{\longrightarrow} H^{d}\left(\hat{G}, \mathbb{I}_{\hat{G}, p}\right) \stackrel{i}{\longrightarrow} \mathbb{Q}_{p} / \mathbb{Z}_{p},
$$

where $X^{\prime}:=\operatorname{Hom}\left(X, \mathbb{I}_{\hat{G}, p}\right), e v_{X}: X^{\prime} \times X \rightarrow \mathbb{I}_{\hat{G}, p}$ is the evaluation map and $i$ is given as in [12, §I.3.5].

The $p$-Poincaré duality group $\hat{G}$ of dimension $d$ is called orientable, if $\mathbb{I}_{\hat{G}, p}$ is a trivial $\hat{G}$-module, and weakly-orientable, if the socle of $\mathbb{I}_{\hat{G}, p}$ is a trivial $\hat{G}$-module, i.e., $\operatorname{soc}\left(\mathbb{I}_{\hat{G}, p}\right) \simeq \mathbb{F}_{p}$.

One can charcterize these groups by continuous cochain cohomology as introduced by J.Tate (cf. [14]) with coefficients in $\mathbb{F}_{p} \llbracket \hat{G} \rrbracket$ as follows:

Proposition 4.2. Let $\hat{G}$ be a profinite group of cohomological p-dimension $d, d \in$ $\mathbb{N}$, and assume (4.3) holds for every finite discrete left $\hat{G}$-module of p-power order $X$. Then the following are equivalent:

(i) $\hat{G}$ is a weakly-orientable p-Poincaré duality group of dimension d,

$$
\mathbf{H}^{k}\left(\hat{G}, \mathbb{F}_{p} \llbracket \hat{G} \rrbracket\right)= \begin{cases}\mathbb{F}_{p} & \text { for } k=d, \\ 0 & \text { for } k \neq d,\end{cases}
$$

where $\mathbb{F}_{p}$ denotes the trivial $\hat{G}$-module and $\mathbf{H}^{\bullet}$ denotes continuous cochain cohomology.

Proof. The implication $(i) \Rightarrow(i i)$ is implicitly already contained in a letter from J.Tate to J-P.Serre (cf. [12, App.1]) Here one should only note that the second property of a Poincaré duality group ensures that $\mathbf{H}^{k}\left(\tilde{G}, \mathbb{F}_{p} \llbracket \tilde{G} \rrbracket\right)^{*}=E_{k}\left(\mathbb{F}_{p}\right)$.

Note that property (4.5) already implies that (4.4) holds for all finite $\mathbb{F}_{p^{-}}$-vector spaces which are discrete $\hat{G}$-modules. Then the same argument used in the proof of [12, Prop.I.32]) shows that (4.4) holds for all finite discrete $\hat{G}$-modules of $p$ power order.

4.3. Cohomological Mackey functors for $p$-Frattini extensions. Let $\mathbf{X}$ be a cohomological $\mathcal{F}(\tilde{G})$-Mackey functor, such that $\mathbf{X}_{U}$ are finitely generated $\mathbb{F}_{p}[\tilde{G} / U]$ modules for all $U \in \mathcal{F}(\tilde{G})$. Then applying $\operatorname{Hom}_{\tilde{G}}\left(-, \mathbb{F}_{p}\right)$ and changing the role of $i$ and $N$ defines a new cohomological $\mathcal{F}(\tilde{G})$-Mackey functor which we denote by $\mathbf{X}^{*}$. The functor ${ }^{*}$ is obviously contravariant and exact.

For short put $\mathbf{S}\left(\mathbb{F}_{p}\right):=\mathfrak{X}\left(\mathbb{F}_{p}\right), \mathbf{T}\left(\mathbb{F}_{p}\right):=\mathbf{S}\left(\mathbb{F}_{p}\right)^{*}$. Then $\mathbf{S}\left(\mathbb{F}_{p}\right)$ is a cohomological $\mathcal{F}(\tilde{G})$-Mackey functor with all mapping $N_{V, U}^{\mathbf{S}\left(\mathbb{F}_{p}\right)}$ bijective, and $\mathbf{T}\left(\mathbb{F}_{p}\right)$ is a $\mathcal{F}(\tilde{G})$ Mackey functor with all mapping $i_{U, V}^{\mathbf{T}\left(\mathbb{F}_{p}\right)}$ bijective, $U, V \in \mathcal{F}(\tilde{G}), V \leq U$.

Thus one has an exact sequence of cohomological $\mathcal{F}(\tilde{G})$-Macke functors

$$
0 \longrightarrow \mathbf{T}\left(\mathbb{F}_{p}\right) \stackrel{\mathfrak{X}\left(\varepsilon^{/ p}\right)^{*}}{\longrightarrow} \mathfrak{X}\left(Q_{0}\right)^{*} \stackrel{\mathfrak{X}\left(\delta^{/ p}\right)^{*}}{\longrightarrow} \mathfrak{X}\left(Q_{1}\right)^{*} .
$$

We put

$$
\begin{aligned}
\Omega^{1}\left(\tilde{G} / \_, \mathbb{F}_{p}\right): & =\operatorname{ker}\left(\mathfrak{X}\left(\delta^{/ p}\right)^{*}\right), \\
\Omega^{2}\left(\tilde{G} / \_, \mathbb{F}_{p}\right): & =\operatorname{coker}\left(\mathfrak{X}\left(\delta^{\prime p}\right)^{*}\right) .
\end{aligned}
$$


TH. WEIGEL

It is an easy exercise to show that $\Omega^{1}\left(\tilde{G} / \ldots, \mathbb{F}_{p}\right)$ is $i$-injective and $N$-surjective, and that $\Omega^{2}\left(\tilde{G} / \ldots, \mathbb{F}_{p}\right)$ is of type $H_{0}$.

4.4. Extending injective maps $\Omega^{1}\left(G, \mathbb{F}_{p}\right) \rightarrow \Omega_{2}\left(G, \mathbb{F}_{p}\right)$. The first step in proving Theorem B is establishing the following proposition:

Proposition 4.3. Let $G$ be a finite group, and let $\alpha: \Omega^{1}\left(G, \mathbb{F}_{p}\right) \rightarrow \Omega^{2}\left(G, \mathbb{F}_{p}\right)$ be a mapping of $\mathbb{F}_{p}[G]$-modules. Then there exists a closed normal subgroup $N$, $N \leq \operatorname{ker}\left(\pi_{p}\right)$ of the universal $p$-Frattini extension $\tilde{G}_{p}, \tilde{G}:=\tilde{G}_{p} / N$, and a map of cohomological $\mathcal{F}(\tilde{G})$-Mackey functors

$$
\boldsymbol{\alpha}: \boldsymbol{\Omega}^{1}\left(\tilde{G} / \longrightarrow, \mathbb{F}_{p}\right) \longrightarrow \mathbf{C}^{/ p},
$$

satisfying im $(\boldsymbol{\alpha})=\mathfrak{N}^{/ p}$ and $\boldsymbol{\alpha}_{\text {ker }\left(\pi_{p}\right)}=\iota_{\text {ker }\left(\pi_{p}\right)}: \alpha$, where $\iota: \mathfrak{N}^{/ p} \rightarrow \mathbf{C}^{/ p}$ denotes the canonical map.

Moreover, if $\alpha$ is injective, $\boldsymbol{\alpha}$ is injective.

Proof. Put $V_{0}:=\operatorname{ker}\left(\pi_{p}\right)$ and $\boldsymbol{\alpha}_{0}:=\alpha: \Omega^{1}\left(G, \mathbb{F}_{p}\right) \rightarrow \Omega_{2}\left(G, \mathbb{F}_{p}\right)$. Assume we have constructed open normal subgroups $V_{0}, . ., V_{k-1}$ and injective morphisms

$$
\boldsymbol{\alpha}_{V_{i}}: \Omega^{1}\left(\tilde{G}_{p} / V_{i}\right) \longrightarrow \Omega_{2}\left(\tilde{G}_{p} / V_{i}, \mathbb{F}_{p}\right)
$$

$i=0, \ldots, k-1$, such that the diagrams

$$
\begin{array}{crr}
\Omega^{1}\left(\tilde{G}_{p} / V_{i-1}, \mathbb{F}_{p}\right) \stackrel{\boldsymbol{\alpha}_{V_{i-1}}}{\longrightarrow} & \Omega_{2}\left(\tilde{G}_{p} / V_{i-1}, \mathbb{F}_{p}\right) \\
i_{V_{i-1}, V_{i}}^{\Omega^{1}} \downarrow & & i_{V_{i-1}, V_{i}}^{\Omega_{2}} \\
\Omega^{1}\left(\tilde{G}_{p} / V_{i}, \mathbb{F}_{p}\right) & \stackrel{\boldsymbol{\alpha}_{V_{i}}}{\longrightarrow} & \Omega_{2}\left(\tilde{G}_{p} / V_{i}, \mathbb{F}_{p}\right) \\
\Omega^{1}\left(\tilde{G}_{p} / V_{i-1}, \mathbb{F}_{p}\right) \stackrel{\boldsymbol{\alpha}_{V_{i-1}}}{\longrightarrow} & \Omega_{2}\left(\tilde{G}_{p} / V_{i-1}, \mathbb{F}_{p}\right) \\
N_{V_{i}, V_{i-1}}^{\Omega^{1}} \uparrow & & \uparrow N_{V_{i}, V_{i-1}}^{\Omega_{2}} \\
\Omega^{1}\left(\tilde{G}_{p} / V_{i}, \mathbb{F}_{p}\right) \stackrel{\boldsymbol{\alpha}_{V_{i}}}{\longrightarrow} & \Omega_{2}\left(\tilde{G}_{p} / V_{i}, \mathbb{F}_{p}\right)
\end{array}
$$

commute, $i=1, . ., k-1$. In the first step we construct $V_{k}$ and a mapping

$$
\boldsymbol{\alpha}_{V_{k}}: \Omega^{1}\left(\tilde{G}_{p} / V_{k}, \mathbb{F}_{p}\right) \rightarrow \Omega_{2}\left(\tilde{G}_{p} / V_{k}, \mathbb{F}_{p}\right)
$$

such the diagrams (4.10) and (4.11) commute for $(k-1, k)$.

Let $V_{k} \leq k \operatorname{er}\left(\pi_{p}\right)$ be the unique open normal subgroup such that $V_{k-1} / V_{k}$ is elementary $p$-abelian, and $i m\left(\boldsymbol{\alpha}_{V_{k-1}}\right)=i m\left(N_{V_{k}, V_{k-1}}^{\Omega_{2}}\right)$. The uniqueness is guaranteed by axiom (iii) of a $/ p$-class field theory. Since $\left(Q_{0}\right)_{V_{k}}^{*}$ is a projective $\mathbb{F}_{p}\left[\tilde{G}_{p} / V_{k}\right]$ module, there exists a mapping $\alpha^{\prime}:\left(Q_{0}\right)_{V_{k}}^{*} \rightarrow \Omega_{2}\left(\tilde{G}_{p} / V_{k}, \mathbb{F}_{p}\right)$ making the diagram

$$
\begin{array}{cc}
\Omega^{1}\left(\tilde{G}_{p} / V_{k-1}, \mathbb{F}_{p}\right) \stackrel{\alpha_{V_{k-1}}}{\longrightarrow} & \Omega_{2}\left(\tilde{G}_{p} / V_{k-1}, \mathbb{F}_{p}\right) \\
N \uparrow & \uparrow N_{V_{k}, V_{k-1}}^{\Omega_{2}} \\
\left(Q_{0}\right)_{V_{k}}^{*} \stackrel{\alpha^{\prime}}{\longrightarrow} & \Omega_{2}\left(\tilde{G}_{p} / V_{k}, \mathbb{F}_{p}\right)
\end{array}
$$

commute, where $N:\left(Q_{0}\right)_{V_{k}}^{*} \rightarrow \Omega^{1}\left(\tilde{G}_{p} / V_{k-1}, \mathbb{F}_{p}\right)$ is the canonical map. Since the $\mathbb{F}_{p}\left[\tilde{G}_{p} / V_{k}\right]$-module $\Omega_{2}\left(\tilde{G}_{p} / V_{k}, \mathbb{F}_{p}\right)$ is directly indecomposable, and as $\left(Q_{0}\right)_{V_{k}}^{*}$ is also injective, $\alpha^{\prime}$ cannot be injective. Hence $\alpha^{\prime}$ factors through a mapping

$$
\boldsymbol{\alpha}_{V_{k}}: \Omega^{1}\left(\tilde{G}_{p} / V_{k}, \mathbb{F}_{p}\right) \rightarrow \Omega_{2}\left(\tilde{G}_{p} / V_{k}, \mathbb{F}_{p}\right) .
$$


for which diagram (4.11) commutes for $(k-1, k)$.

Let $x \in \Omega^{1}\left(\tilde{G}_{p} / V_{k-1}, \mathbb{F}_{p}\right)$. As $\Omega^{1}\left(\tilde{G}_{p} / \ldots, \mathbb{F}_{p}\right)$ is $N$-surjective, there exists $y \in$ $\Omega^{1}\left(\tilde{G}_{p} / V_{k}, \mathbb{F}_{p}\right)$ such that $N_{V_{k}, V_{k-1}}^{\Omega^{1}}(y)=x$. Thus

$$
\begin{aligned}
i_{V_{k-1}, V_{k}}^{\Omega_{2}}\left(\boldsymbol{\alpha}_{V_{k-1}}(x)\right) & =i_{V_{k-1}, V_{k}}^{\Omega_{2}}\left(\boldsymbol{\alpha}_{V_{k-1}}\left(N_{V_{k}, V_{k-1}}^{\Omega^{1}}(y)\right)\right), \\
& =i_{V_{k-1}, V_{k}}^{\Omega_{2}}\left(N_{V_{k}, V_{k-1}}^{\Omega_{2}}\left(\boldsymbol{\alpha}_{V_{k}}(y)\right)\right)=N_{V_{k-1} / V_{k}}\left(\boldsymbol{\alpha}_{V_{k}}(y)\right),
\end{aligned}
$$

where $N_{V_{k-1} / V_{k}}:=\sum_{g \in V_{k-1} / V_{k}} g$. On the other hand

$$
\begin{aligned}
\boldsymbol{\alpha}_{V_{k}}\left(i_{V_{k-1}, V_{k}}^{\Omega^{1}}(x)\right) & =\boldsymbol{\alpha}_{V_{k}}\left(i_{V_{k-1}, V_{k}}^{\Omega^{1}}\left(N_{V_{k}, V_{k-1}}^{\Omega^{1}}(y)\right)\right) \\
& =\boldsymbol{\alpha}_{V_{k}}\left(N_{V_{k-1} / V_{k}}(y)\right)=N_{V_{k-1} / V_{k}}\left(\boldsymbol{\alpha}_{V_{k}}(y)\right),
\end{aligned}
$$

i.e., the diagram (4.10) commutes for $(k-1, k)$ aswell.

Since $i_{V_{k-1}, V_{k}}^{1}: \operatorname{soc}\left(\Omega^{1}\left(\tilde{G}_{p} / V_{k-1}, \mathbb{F}_{p}\right) \rightarrow \operatorname{soc}\left(\Omega^{1}\left(\tilde{G}_{p} / V_{k}, \mathbb{F}_{p}\right)\right.\right.$ is bijective, and as $\mathbf{C}^{/ p}$ is of type $H^{0}, \boldsymbol{\alpha}_{V_{k}}$ is injective provided $\boldsymbol{\alpha}_{V_{k-1}}$ is injective.

Let $N$ : $=\bigcap_{k \in \mathbb{N}_{0}} V_{k}$. Then $\left\{V_{k} / N\right\}_{k \in \mathbb{N}_{0}}$ is a basis of open neighbourhoods of $1 \in \tilde{G}_{p} / N$.

Let $V \in \mathcal{F}_{N}:=\{U \in \mathcal{F} \mid N \leq U\}$. Then there exist $k \in \mathbb{N}_{0}$ such that $V_{k} \leq V$. Since $\Omega^{1}\left(\tilde{G}_{p} / \ldots, \mathbb{F}_{p}\right)$ and $\Omega_{2}\left(\tilde{G}_{p} /,, \mathbb{F}_{p}\right)$ are $i$-injective cohomological $\mathcal{F}$ Mackey functors, there exists a unique mapping

$$
\boldsymbol{\alpha}_{V}: \Omega^{1}\left(\tilde{G}_{p} / V, \mathbb{F}_{p}\right) \longrightarrow \Omega_{2}\left(\tilde{G}_{p} / V, \mathbb{F}_{p}\right)
$$

making the diagram

$$
\begin{array}{crc}
\Omega^{1}\left(\tilde{G}_{p} / V, \mathbb{F}_{p}\right) \stackrel{\boldsymbol{\alpha}_{V}}{\longrightarrow} & \Omega_{2}\left(\tilde{G}_{p} / V, \mathbb{F}_{p}\right) \\
i_{V, V_{k}}^{\Omega^{1}} \downarrow & \downarrow i_{V, V_{k}}^{\Omega_{2}} \\
\Omega^{1}\left(\tilde{G}_{p} / V_{k}, \mathbb{F}_{p}\right) \stackrel{\boldsymbol{\alpha}_{V_{k}}}{\longrightarrow} \Omega_{2}\left(\tilde{G}_{p} / V_{k}, \mathbb{F}_{p}\right)
\end{array}
$$

commute. It is easy to check that for all $U, V \in \mathcal{F}_{N}, V \leq U$, the diagram

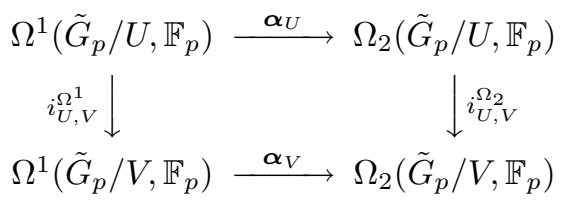

commutes. Note that $\Omega_{2}\left(\tilde{G} / \ldots, \mathbb{F}_{p}\right)$ is $i$-injective, and that for $x \in \Omega^{1}\left(\tilde{G}_{p} / V, \mathbb{F}_{p}\right)$

$$
\begin{aligned}
& i_{U, V}^{\Omega_{2}}\left(\boldsymbol{\alpha}_{U}\left(N_{V, U}^{\Omega^{1}}(x)\right)\right)=\boldsymbol{\alpha}_{V}\left(i_{U, V}^{\Omega^{1}}\left(N_{V, U}^{\Omega^{1}}(x)\right)\right)=\boldsymbol{\alpha}_{V}\left(N_{U / V}(x)\right), \\
& i_{U, V}^{\Omega_{2}}\left(N_{V, U}^{\Omega_{2}}\left(\boldsymbol{\alpha}_{V}(x)\right)\right)=N_{V / U}\left(\boldsymbol{\alpha}_{V}(x)\right)=\boldsymbol{\alpha}_{V}\left(N_{U / V}(x)\right) .
\end{aligned}
$$

Hence the diagram

$$
\begin{array}{cr}
\Omega^{1}\left(\tilde{G}_{p} / U, \mathbb{F}_{p}\right) \stackrel{\alpha_{U}}{\longrightarrow} & \Omega_{2}\left(\tilde{G}_{p} / U, \mathbb{F}_{p}\right) \\
N_{V, U}^{\Omega^{1}} \uparrow & \uparrow N_{V, U}^{\Omega_{2}} \\
\Omega^{1}\left(\tilde{G}_{p} / V, \mathbb{F}_{p}\right) \stackrel{\boldsymbol{\alpha}_{V}}{\longrightarrow} \Omega_{2}\left(\tilde{G}_{p} / V, \mathbb{F}_{p}\right)
\end{array}
$$

commutes as well showing that

$$
\boldsymbol{\alpha}: \Omega^{1}\left(\tilde{G}_{p} / \ldots, \mathbb{F}_{p}\right) \longrightarrow \Omega_{2}\left(\tilde{G}_{p} / \ldots, \mathbb{F}_{p}\right)
$$


is a morphism of cohomological $\mathcal{F}\left(\tilde{G}_{p} / N\right)$-Mackey functors. By construction, one has $i m(\boldsymbol{\alpha})=\mathfrak{N} / p$. Moreover, if $\alpha$ is injective, then the construction shows that $\boldsymbol{\alpha}$ is also injective. This yields the claim.

4.5. $\Omega^{1}$-relator $p$-Frattini extensions. Let $\pi: \tilde{G} \rightarrow G$ be a $p$-Frattini extension of $G$, and let $\left(\mathbf{C}^{/ p}, \gamma^{/ p}\right)$ denote its $/ p$-Frattini class field theory. We call $\pi$ an $\Omega^{1}$-relator $p$-Frattini extension, if there exists a map

$$
\boldsymbol{\alpha}: \Omega^{1}\left(\tilde{G} /-, \mathbb{F}_{p}\right) \rightarrow \mathbf{C}^{/ p}
$$

of cohomological $\mathcal{F}(\tilde{G})$-Mackey functors with $i m(\alpha)=\mathfrak{N} / p$. If necessary we include the mapping $\boldsymbol{\alpha}$ in the notation, i.e., we write $(\pi, \alpha)$ for a $\Omega^{1}$-relator $p$-Frattini extension.

For the universal $p$-Frattini extension $\pi_{p}: \tilde{G}_{p} \rightarrow G$ one has $\mathfrak{N}^{/ p}=0$, and thus $\pi_{p}$ is a $\Omega^{1}$-relator $p$-Frattini extension.

From Proposition 4.3 one concludes that one can also construct such a $p$-Frattini extenion starting from a map $\alpha: \Omega^{1}\left(G, \mathbb{F}_{p}\right) \rightarrow \Omega_{2}\left(G, \mathbb{F}_{p}\right)$.

Another source of examples arises in the context of modular towers. The starting point in the study of modular towers is a fixed surjective morphism $\phi: \hat{G} \rightarrow G$ where $\hat{G}$ is a certain profinite orientable $p$-Poincaré duality group of dimension 2 onto a finite group $G$. A modular tower consists of all open normal subgroups $U$ in $\hat{G}$ contained in $\operatorname{ker}(\phi)$ such that the induced map $\phi_{U}: \tilde{G} / U \rightarrow G$ is a $p$ Frattini extension (cf. [1]). The 'limit groups' of a modular tower correspond to a closed normal subgroup $A \leq \operatorname{ker}(\phi)$ such that $\phi_{A}: \hat{G} / A \rightarrow G$ is a maximal $p$ Frattini extension $\phi$ can factor through. In particular, $\left(\phi_{A}, \pi_{A}\right), \pi_{A}: \tilde{G} \rightarrow \hat{G} / A$ the canonical projection, is a maximal p-Frattini quotient of $\phi$ (cf. [16]). These $p$-Frattini extension have the following property.

Proposition 4.4. Let $\phi: \hat{G} \rightarrow G$ be a surjective map of the profinite weaklyorientable p-Poincaré duality group $\hat{G}$ of dimension 2 onto the finite group $G$. Then for every maximal p-Frattini quotient $(\pi, \beta), \pi: i m(\beta) \rightarrow G$ is a $\Omega^{1}$-relator $p$ Frattini extension of $G$.

Proof. Let $B:=i m(\beta)$, and let

$$
Q_{1} \stackrel{\delta^{p}}{\longrightarrow} Q_{0} \longrightarrow \mathbb{F}_{p}
$$

be a partial minimal projective resolution in ${ }_{B} \operatorname{prf}_{/ p}$. Put $M:=k e r(\delta)$. By $[16$, Prop.3.4], one has a surjective map $\alpha: Q_{0} \rightarrow M$. Since $\mathfrak{N}^{/ p}$ is norm surjective (cf. Prop.4.1(b)), one has a surjective map of cohomological $\mathcal{F}(B)$-Mackey functors

$$
\rho: \mathfrak{X}\left(Q_{0}\right) \longrightarrow \mathfrak{X}(M) \longrightarrow \mathfrak{N}^{/ p} .
$$

Since $\mathfrak{N} / p$ is a $\mathcal{F}(B)$-sub Mackey functor of $\mathbf{C}$, and as $\left(Q_{0}\right)_{U}$ is an injective $\mathbb{F}_{p}[B / U]$ module, $\rho_{U}:\left(Q_{0}\right)_{U} \rightarrow \mathfrak{N}_{U}^{/ p} \leq \Omega_{2}\left(B / U, \mathbb{F}_{p}\right)$ cannot be injective, i.e, $\operatorname{soc}\left(\left(Q_{0}\right)_{U}\right) \leq$ $\operatorname{ker}\left(\rho_{U}\right)$. Hence $\rho$ induces a surjective mapping

$$
\rho_{*}: \Omega^{1}\left(B / \_, \mathbb{F}_{p}\right) \longrightarrow \mathfrak{N}^{/ p}
$$

of cohomological $\mathcal{F}(B)$-Mackey functors and this yields the claim.

In order to finish the proof of Theorem B, we establish the following theorem: 
Theorem 4.5. Let $(\pi, \boldsymbol{\alpha}), \pi: \tilde{G} \rightarrow G$, be a $\Omega^{1}$-relator $p$-Frattini extension. Assume further that $\boldsymbol{\alpha}$ is injective, and that $\boldsymbol{\alpha}_{k e r(\pi)}$ is not an isomorphism. Then $\tilde{G}$ is a weakly-orientable p-Poincaré duality group of dimension 2.

Proof. Note that $\operatorname{dim}_{\mathbb{F}_{p}}\left(\Omega_{2}\left(G, \mathbb{F}_{p}\right)\right)>\operatorname{dim}_{\mathbb{F}_{p}}\left(\Omega^{1}\left(G, \mathbb{F}_{p}\right)\right)$ implies that $\tilde{G}$ is infinite (cf. [16, Prop.3.5]). It suffices to prove that $\mathbf{H}^{k}\left(\tilde{G}, \mathbb{F}_{p} \llbracket \tilde{G} \rrbracket\right)=0$ for $k \neq 2$, and $\mathbf{H}^{2}\left(\tilde{G}, \mathbb{F}_{p} \llbracket \tilde{G} \rrbracket\right) \simeq \mathbb{F}_{p}$. As beforre $\mathbf{H}^{\bullet}$ denotes continuous cochain cohomology.

By definition, one has exact sequences of cohomological $\mathcal{F}(\tilde{G})$-Mackey functors

$$
\begin{gathered}
0 \longrightarrow \mathbf{T}\left(\mathbb{F}_{p}\right) \longrightarrow \mathfrak{X}\left(Q_{0}\right) \longrightarrow \Omega^{1}\left(\tilde{G} / \longrightarrow, \mathbb{F}_{p}\right) \longrightarrow 0, \\
0 \longrightarrow \Omega^{1}\left(\tilde{G} /-, \mathbb{F}_{p}\right) \longrightarrow \Omega_{2}\left(\tilde{G} /-, \mathbb{F}_{p}\right) \longrightarrow \mathbf{A b}^{/ p} \longrightarrow 0, \\
0 \longrightarrow \Omega_{2}\left(\tilde{G} /-, \mathbb{F}_{p}\right) \longrightarrow \mathfrak{X}\left(Q_{1}\right) \longrightarrow \mathfrak{X}\left(Q_{0}\right) \longrightarrow \mathbf{S}\left(\mathbb{F}_{p}\right) \longrightarrow 0 .
\end{gathered}
$$

As $\tilde{G}$ is infinite $m\left(\mathbf{T}\left(\mathbb{F}_{p}\right)\right)=m\left(\mathbf{A b}^{/ p}\right)=0$. Thus applying the functor $m$ yields that one has a minimal projective resolution

$$
0 \longrightarrow Q_{0} \longrightarrow Q_{1} \longrightarrow Q_{0} \longrightarrow \mathbb{F}_{p} \longrightarrow 0
$$

of $\mathbb{F}_{p}$ in ${ }_{\tilde{G}} \mathbf{p r f}{ }_{/ p}$. Hence $\tilde{G}$ is of cohomological $p$-dimension 2 .

In his letter to J-P.Serre (cf. [12, App.1]), J.Tate described how one can compute the Pontryagin dual of the cohomology groups $\mathbf{H}^{k}\left(\tilde{G}, \mathbb{F}_{p} \llbracket \tilde{G} \rrbracket\right)$. Translated to our situation we obtain

$$
\begin{aligned}
& \mathbf{H}^{2}\left(\tilde{G}, \mathbb{F}_{p} \llbracket \tilde{G} \rrbracket\right)^{*}=\underset{U}{\lim _{U}} \mathbf{H}_{2}\left(U, \mathbb{F}_{p}\right), \\
& \mathbf{H}^{1}\left(\tilde{G}, \mathbb{F}_{p} \llbracket \tilde{G} \rrbracket\right)^{*}=\underset{U}{\lim _{\longrightarrow}} \mathbf{H}_{1}\left(U, \mathbb{F}_{p}\right) .
\end{aligned}
$$

Since $\tilde{G}$ is infinite, $\mathbf{H}^{0}\left(\tilde{G}, \mathbb{F}_{p} \llbracket \tilde{G} \rrbracket\right)=0$. From the exact sequences (4.28) it follows that one has an isomorphism of $\mathcal{F}(\tilde{G})$-Mackey functors $\mathbf{H}_{2}\left(\ldots, \mathbb{F}_{p}\right) \simeq \mathbf{T}\left(\mathbb{F}_{q}\right)$. This yields $\mathbf{H}^{2}\left(\tilde{G}, \mathbb{F}_{p} \llbracket \tilde{G} \rrbracket\right) \simeq \mathbb{F}_{p}$.

Let $\boldsymbol{\alpha}^{*}: \Omega^{2}\left(\tilde{G} / \ldots, \mathbb{F}_{p}\right) \longrightarrow \Omega_{1}\left(\tilde{G} / \ldots, \mathbb{F}_{p}\right)$ be the Pontryagin dual of $\boldsymbol{\alpha}$. Then by (4.32), $\mathbf{H}^{1}\left(\tilde{G}, \mathbb{F}_{p} \llbracket \tilde{G} \rrbracket\right) \simeq m\left(k e r\left(\boldsymbol{\alpha}^{*}\right)\right)$. Moreover, $\boldsymbol{\alpha}^{*}$ is surjective. Since for all $U \in \mathcal{F}(\tilde{G})$, one has an isomorphism

$$
h d\left(\boldsymbol{\alpha}_{U}^{*}\right): h d\left(\Omega_{2}\left(\tilde{G} / U, \mathbb{F}_{p}\right)\right) \longrightarrow h d\left(\Omega_{1}\left(\tilde{G} / U, \mathbb{F}_{p}\right)\right),
$$

where $h d(-)$ denotes the head of a module, one obtains a commutative diagram

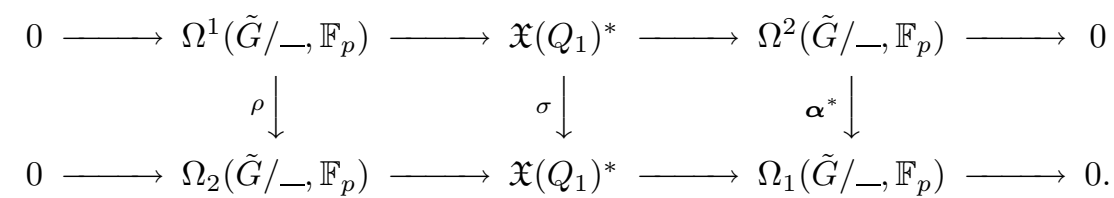

By (4.33), $\sigma$ is an isomorphism. So by the snake lemma, $\rho$ is injective, and one has an isomorphism coker $(\rho)=\operatorname{ker}\left(\boldsymbol{\alpha}^{*}\right)$. Since $\Omega^{1}\left(\tilde{G} / \ldots, \mathbb{F}_{p}\right)$ is $N$-surjective, all elements in $\operatorname{im}(\sigma)$ are universal norms. Hence by dimension arguments, $\operatorname{im}(\rho)=$ $i m(\boldsymbol{\alpha})$ and this yields

$$
m\left(k e r\left(\boldsymbol{\alpha}^{*}\right)\right) \simeq m(\operatorname{coker}(\rho)) \simeq m\left(\mathbf{A} \mathbf{b}^{/ p}\right)=0 .
$$

This yields the claim.

Corollary 4.6. Let $G$ be a finite group and let $p$ be a prime number. Then the following are equivalent: 
(i) There exists a p-Frattini extension $\pi: \tilde{G} \rightarrow G$ with $\tilde{G}$ a profinite weaklyorientable p-Poincaré duality group of dimension 2.

(ii) There exists an injection $\alpha: \Omega^{1}\left(G, \mathbb{F}_{p}\right) \rightarrow \Omega_{2}\left(\mathbb{F}_{p}\right)$ which is not an isomorphism.

Proof. This is a direct consequence of [16, Thm.4.1] and Theorem 4.5.

Remark 4.7. (a) Let $p=2$ and let $G=P S l_{2}(q), q \equiv 3 \bmod 4$. The explicit description of the projective indecomposable $\mathbb{F}_{2}[G]$-modules obtained by K.Erdmann $[5]$ shows that in this case one has an injection $\alpha: \Omega^{1}\left(G, \mathbb{F}_{p}\right) \rightarrow \Omega_{2}\left(G, \mathbb{F}_{p}\right)$.

(b) If $G$ is $p$-perfect, i.e., $G_{p}^{a b}=0, \tilde{G}$ is $p$-perfect too. Thus every $\tilde{G}$-module $M \in o b\left({ }_{\tilde{G}} \mathbf{p r f}_{p}\right)$, which underlying abelian pro- $p$ group is isomorphic to $\mathbb{Z}_{p}$ and which reduction $\bmod p M / p . M$ is a trivial $\tilde{G}$-module, must be trivial. Hence in this case one can conclude that $\tilde{G}$ is indeed a orientable $p$-Poincaré duality group of dimension 2.

(c) In [16, Ex.1.4] an example was given were for any maximal $p$-Frattini quotient $(\pi, \beta)$ of a morphism $\phi: \hat{G} \rightarrow P S l_{2}(7)$, the $p$-Frattini extension $\pi$ is of the type described in Theorem 4.5.

(d) One question which has been untouched completely is to describe all isomorphism types of extensions $\pi: \tilde{G} \rightarrow G$ satisfying (i) of Corollary 4.6. The construction we used does not give any evidence how one can achieve this goal.

\section{5. $\Delta$-Frattini EXTENSIONS}

Throughout this section we fix a prime number $p$. For a given finite group $G$ we denote by $\mathfrak{S}_{p}(G)$ the set of isomorphism types of irreducible (left) $\mathbb{F}_{p}[G]$-modules. For an irreducible $\mathbb{F}_{p}[G]$-module $S$ we use the symbol $[S] \in \mathfrak{S}_{p}(G)$ to denote its isomorphism type.

5.1. The $\Delta$-head of an $\mathbb{F}_{p}[G]$-module. Let $\Delta \subseteq \mathfrak{S}_{p}(G)$ be a set of isomorphism types of irreducible $\mathbb{F}_{p}[G]$-modules. For short we call an $\mathbb{F}_{p}[G]$-module $M \in o b\left({ }_{G} \bmod _{p}\right)$ of finite $\mathbb{F}_{p}$-dimension a $\Delta$-module, if $M$ has a composition series $\left(M_{k}\right)_{0 \leq k \leq m}, 0=M_{0}<M_{1}<\cdots<M_{m}=M$, with each composition factor being contained in $\Delta$, i.e., $\left[M_{k} / M_{k-1}\right] \in \Delta$ for all $k=1, . ., m$. We also assume that $0 \in o b\left({ }_{G} \bmod _{p}\right)$ is a $\Delta$-module.

Let $M$ be an $\mathbb{F}_{p}[G]$-module of finite $\mathbb{F}_{p}$-dimension. We call an $\mathbb{F}_{p}[G]$-submodule $N \leq M$ a $\Delta$-kernel, if $M / N$ is a $\Delta$-module. Obviously, the intersection of any set of $\Delta$-kernels $N_{i} \leq M, i \in I$, is again a $\Delta$-kernel. Hence there exists a minimal $\Delta$-kernel $M_{\Delta} \leq M$. For short we call

$$
h d_{\Delta}(M):=M / M_{\Delta}
$$

The $\Delta$-head of $M$.

\subsection{The universal $\Delta$-Frattini extension. Let}

$$
1 \longrightarrow \Omega_{2}\left(G, \mathbb{F}_{p}\right) \stackrel{\iota}{\longrightarrow} \tilde{G}_{/ p} \stackrel{\pi_{/ p}}{\longrightarrow} G \longrightarrow 1
$$

be the universal elementary $p$-abelian Frattini extension of $G$, where $\iota$ is considered to be given by inclusion. Factoring by the minimal $\Delta$-kernel $\Omega_{2}\left(G, \mathbb{F}_{p}\right)_{\Delta}$ of $\Omega_{2}\left(G, \mathbb{F}_{p}\right)$ yields a $\Delta$-Frattini extension

$$
1 \longrightarrow h d\left(\Omega_{2}\left(G, \mathbb{F}_{p}\right)\right) \stackrel{\iota}{\longrightarrow} \tilde{G}_{/ \Delta} \stackrel{\pi_{/ \Delta}}{\longrightarrow} G \longrightarrow 1
$$


which is easily seen to be universal with respect to all elementary $p$-abelian $\Delta$ Frattini extensions of $G$. Thus for $G_{0}:=G$, and $\pi_{i+1, i}: G_{i+1} \rightarrow G_{i}$ the universal elementary $p$-abelian $\Delta$-Frattini extension of $G_{i}$, we obtain an inverse system whose inverse limit

$$
\tilde{G}_{\Delta}:=\lim _{i \in \mathbb{N}_{0}} G_{i}
$$

together with the canonical map $\pi_{\Delta}: \tilde{G}_{\Delta} \rightarrow G$ is a $\Delta$-Frattini extension of $G$. The universality aswell as the uniqueness up to isomorphism follows by the same arguments which were used to prove these statements for the universal $p$-Frattini extension (cf. [6]).

At this point we have to deal with the question how one characterize the universal $\Delta$-Frattini extension among all $\Delta$-Frattini extensions. This is the subject of the following proposition.

Proposition 5.1. Let $\pi: \tilde{G} \rightarrow G$ be a $\Delta$-Frattini extension of $G, \Delta \subseteq \mathfrak{S}_{p}(G)$. Then the following are equivalent:

(i) $\pi$ coincides with the universal $\Delta$-Frattini extension of $G$.

(ii) $H^{2}(\tilde{G}, S)=0$ for all irreducible $\mathbb{F}_{p}[G]$-modules $S,[S] \in \Delta$.

Proof. Assume that $\pi: \tilde{G} \rightarrow G$ is the universal $\Delta$-Frattini extension of $G$, and that there exists an irreducible $\mathbb{F}_{p}[G]$-module $S,[S] \in \Delta$, with $H^{2}(\tilde{G}, S) \neq 0$. For $\eta \in H^{2}(\tilde{G}, S), \eta \neq 0$, the associated extension of profinite groups

$$
\mathbf{s}(\eta): \quad 1 \longrightarrow S \longrightarrow X \stackrel{\tau}{\longrightarrow} \tilde{G} \longrightarrow 1
$$

is non-split and thus $\tau \circ \pi: X \rightarrow G$ is a $\Delta$-Frattini extension. The universality of $\pi$ implies that $\tau$ has a section $\sigma: \tilde{G} \rightarrow X$ contradicting the fact that $\mathbf{s}(\eta)$ is non-split. Thus (i) implies (ii).

Assume that $H^{2}(\tilde{G}, S)=0$ for all $[S] \in \Delta$, and let $\pi_{\Delta}: \tilde{G}_{\Delta} \rightarrow G$ be the universal $\Delta$-Frattini extension of $G$. Then one has a surjective map $\beta$ : $\tilde{G}_{\Delta} \rightarrow \tilde{G}$, and thus an isomorphism

$$
\tilde{\beta}^{-1}: \tilde{G} \longrightarrow \tilde{G}_{\Delta} / \operatorname{ker}(\beta) .
$$

Assume that $\operatorname{ker}(\beta) \neq 1$ is non-trivial, and let $U \leq \operatorname{ker}(\beta)$ be a maximal open subgroup of $\operatorname{ker}(\beta)$ which is normal in $\tilde{G}_{\Delta}$. Since $[\operatorname{ker}(\beta) / U] \in \Delta$, one has $H^{2}(\tilde{G}, \operatorname{ker}(\beta) / U)=0$. Hence the embedding problem

$$
\text { s: } 1 \longrightarrow \operatorname{ker}(\beta) / U \longrightarrow \tilde{G}_{\Delta} / U \longrightarrow \tilde{G}_{\Delta} / \operatorname{ker}(\beta) \longrightarrow 1
$$

has a weak solution (cf. [16, Prop.3.2]). This implies that $\mathbf{s}$ is split exact, which contradicts the fact that $\mathbf{s}$ is also a $p$-Frattini extension. Thus $\operatorname{ker}(\beta)=1$, and this yields the claim.

5.3. Chevalley groups over $\mathbb{Z}_{p}$. For a given Dynkin diagram $D$ let $X_{D}$ be the simple simply-connected $\mathbb{Z}$-Chevalley group scheme associated to $D$, i.e., if $D$ is of type $A_{n}$, one has $X_{D}=S l_{n+1}$. It has been proved in [18, Thm.B] that

$$
\pi_{D}: X_{D}\left(\mathbb{Z}_{p}\right) \longrightarrow X_{D}\left(\mathbb{F}_{p}\right)
$$


is a $p$-Frattini extension apart from possibly 11 explicitly known values of $(D, p)$. It was also shown that in 8 of these 11 cases (5.8) fails to be a $p$-Frattini extension.

In case $\pi_{D}$ is a $p$-Frattini extension, then it is also a $\Delta_{D}$-Frattini extension, where $\Delta_{D}$ consists of all the $\mathbb{F}_{p}\left[X\left(F_{p}\right)\right]$-composition factors of the $\mathbb{F}_{p}$-Chevalley Lie algebra $\mathfrak{L}_{D} \otimes \mathbb{F}_{p}$ (cf. $[18,(2.5)]$ ). If one has additionally

$$
\begin{aligned}
&(D, p) \notin\{\left(A_{n}, p\right), p \mid(n+1),\left(B_{n}, 2\right),\left(C_{n}, 2\right),\left(D_{n}, 2\right), \ldots \\
&\left.\ldots,\left(E_{6}, 3\right),\left(E_{7}, 2\right),\left(F_{4}, 2\right),\left(G_{2}, 2\right),\left(G_{2}, 3\right)\right\},
\end{aligned}
$$

then $\mathfrak{L}_{D} \otimes \mathbb{F}_{p}$ is an irreducible $\mathbb{F}_{p}\left[X_{D}\left(\mathbb{F}_{p}\right)\right]$-module (cf. [18, Lemma 2.10]), and thus $\Delta_{D}=\left\{\left[\mathfrak{L}_{D} \otimes \mathbb{F}_{p}\right]\right\}$.

The question raised in [6, Prob.20.40] can now be restated in the following way.

Question 5.2. Assume that $p$ is large with respect to the Coxeter number of $D$. Is it true that the $p$-Frattini extension $\pi_{D}: X\left(\mathbb{Z}_{p}\right) \rightarrow X\left(\mathbb{F}_{p}\right)$ coincide with the universal $\Delta_{D}$-Frattini extension?

From Proposition 5.1 one concludes that the problem of Question 5.2 is equivalent to the following vanishing problem.

Question 5.3. Assume that $p$ is large with respect to the Coxeter number of D. Is it true that

$$
H^{2}\left(X_{D}\left(\mathbb{Z}_{p}\right), \mathfrak{L}_{D} \otimes \mathbb{F}_{p}\right)=0 ?
$$

As we see in the following theorem both questions have an affirmative answer for $X_{D}=S l_{2}$.

Theorem 5.4. Let $p$ be a prime number different from 2, 3 or 5 . Then

$$
\pi_{A_{1}}: S l_{2}\left(\mathbb{Z}_{p}\right) \rightarrow S l_{2}\left(\mathbb{F}_{p}\right)
$$

coincides with the universal $\Delta$-Frattini extension for all $\Delta \subseteq \mathfrak{S}_{p}\left(\operatorname{Sl}_{2}\left(\mathbb{F}_{p}\right)\right)$ satisfying $\left[M_{2}\right] \in \Delta,\left[M_{p-3}\right] \notin \Delta$, where $M_{k}, k=0, . ., p-1$ denotes the irreducible $\mathbb{F}_{p}\left[S l_{2}\left(\mathbb{F}_{p}\right)\right]$ module of heighest weight $k$ and $\mathbb{F}_{p}$-dimension $k+1$.

Proof. By the previously mentioned remark and Proposition 5.1 it suffices to show that $H^{2}\left(S l_{2}\left(\mathbb{Z}_{p}\right), M_{k}\right)=0$ for all $k \neq p-3$.

As $p \neq 2,3, \tilde{G}:=S l_{2}\left(\mathbb{Z}_{p}\right)$ is $p$-torsionfree, and thus a $p$-Poincaré duality group of dimension $d$ (cf. [13, Prop.4.4.1]). As we assumed $p \neq 2,3, \tilde{G}$ is perfect (cf. [18, Prop.3.2]). Thus its $p$-dualizing module $\mathbb{I}_{\tilde{G}, p}$ is a trivial $\tilde{G}$-module. Hence by Poincaré duality and the Universal Coefficiant Theorem one has

$$
H^{2}\left(S l_{2}\left(\mathbb{Z}_{p}\right), M_{k}\right) \simeq H_{1}\left(S l_{2}\left(\mathbb{Z}_{p}\right), M_{k}\right) \simeq H^{1}\left(S l_{2}\left(Z_{p}\right), M_{k}\right)^{*}
$$

where ${ }^{*}$ denotes the Pontryagin dual. Moreover, from [16, Prop.3.1] and [17] one concludes that

$$
H^{1}\left(S l_{2}\left(\mathbb{Z}_{p}\right), M_{k}\right) \simeq H^{1}\left(S l_{2}\left(\mathbb{F}_{p}\right), M_{k}\right)=0
$$

for $k \neq p-3$. This yields the claim.

Remark 5.5. Theorem 5.4 does not hold for $p=2,3$ or 5 , but in each case for a different reason.

For $p=2$ or $3, \pi_{A_{1}}$ is not a 2-Frattini extension (cf. [18, Thm.B]). For $p=3, \pi_{A_{1}}$ is even a split extension, since in this case $\mathfrak{L}_{A_{1}} \otimes \mathbb{F}_{3}$ is isomorphic to the Steinberg module for $S l_{2}\left(\mathbb{F}_{3}\right)$. 
For $p=5, \Omega_{2}\left(S l_{2}\left(\mathbb{F}_{5}\right), \mathbb{F}_{5}\right)$ is a $\Delta_{A_{1}}$-module (cf. [17]). Hence the universal elementary $p$-abelian $\Delta_{A_{1}}$-extension coincides with the universal elementary $p$-abelian Frattini extension $\pi_{/ p}$. However,

$$
\operatorname{dim}_{\mathbb{F}_{5}}\left(\Omega_{2}\left(S l_{2}\left(\mathbb{F}_{5}\right), \mathbb{F}_{5}\right)=6, \quad \operatorname{dim}_{\mathbb{F}_{5}}\left(\operatorname{ker}\left(\pi_{A_{1}}\right)^{a b}\right)=3 .\right.
$$

This phenomenon can also be explained by analyzing cohomology groups. Since $p-3=2$, Poincaré duality and [16, Prop.3.1] implies that

$$
H^{2}\left(S l_{2}\left(\mathbb{Z}_{5}\right), \mathfrak{L}_{A_{1}} \otimes \mathbb{F}_{5}\right)^{*} \simeq H^{1}\left(S l_{2}\left(\mathbb{Z}_{5}\right), \mathfrak{L}_{A_{1}} \otimes \mathbb{F}_{5}\right) \simeq H^{1}\left(S l_{2}\left(\mathbb{F}_{5}\right), \mathfrak{L}_{A_{1}} \otimes \mathbb{F}_{5}\right) \simeq \mathbb{F}_{5} .
$$

\section{REFERENCES}

[1] P. Bailey and M. D. Fried. Hurwitz monodromy, spin seperation and higher levels of modular towers. Proc. Sympos. Pure Math., 70:79-200, 2000.

[2] A. Brumer. Pseudocompact algebras, profinite groups and class formations. J. Algebra, 4:442470, 1966.

[3] J. Cossey, O. H. Kegel, and L. G. Kovács. Maximal Frattini extensions. Arch. Math. (Basel), $35(3): 210-217,1980$.

[4] A. Dress. Contributions to the theory of induced representations, volume 342 of Lecture Notes in Mathematics. Springer-Verlag, Berlin, 1973.

[5] K. Erdmann. Principal blocks of groups with dihedral sylow 2-subgroups. Comm. Algebra, 5:665-694, 1977.

[6] M. D. Fried and M. Jarden. Field Arithmetic. Ergebnisse der Mathematik und ihrer Grenzgebiete, 3.Folge, Band 11. Springer-Verlag, New York, 1986.

[7] K. W. Gruenberg. Projective profinite groups. J. London Math. Soc., 47:155-165, 1967.

[8] K. W. Gruenberg. Relation modules for finite groups, volume 25 of Conference Board of the Mathematical Sciences Regional Conference Series in Mathematics. American Mathematical Society, Providence, R.I., 1976.

[9] S. Mac Lane. Homology. Classics in Mathematics. Springer-Verlag, Berlin, 1995.

[10] J. Neukirch. Algebraische Zahlentheorie. Springer-Verlag, Berlin, 1992.

[11] L. Ribes and P. Zalesskii. Profinite Groups, volume 40 of Ergebnisse der Mathematik und ihrer Grenzgebiete, 3.Folge. Springer-Verlag, Berlin, 2000.

[12] J-P. Serre. Galois Cohomology. Springer-Verlag, Berlin, cinquième édition, révisée et complétée edition, 1997.

[13] P. Symonds and Th. Weigel. Cohomology of p-adic analytic groups. In M. duSautoy, D. Segal, and A. Shalev, editors, New horizons in pro-p groups, volume 184 of Progress in Mathematics, pages 349-410. Birkhäuser, Boston, 2000.

[14] J. Tate. Relations between $K_{2}$ and Galois cohomology. Invent. Math., 36:257-274, 1976.

[15] P. Webb. User Guide to Mackey Funktors. In M. Hazewinkel, editor, Handbook of Algebra, volume 2, pages 805-836. Elsevier, 2000.

[16] Th. Weigel. Maximal $\ell$-frattini quotients of $\ell$-Poincaré duality groups of dimension 2 . to appear in Arch. Math. (Basel).

[17] Th. Weigel. On the universal Frattini extension of a finite group. submitted.

[18] Th. Weigel. On the Profinite Completion of Arithmetic Groups of Split Type. In M. Goze, editor, Lois d'algèbres et variété algébrique, volume 50 of Travaux en cours, pages 79-101. Hermann, Paris, 1996.

Th. Weigel, Università di Milano-Bicocca, U5-3067, Via R.Cozzi, 53, 20125 Milano, ITALY

E-mail address: thomas.weigel@unimib.it 